\title{
Two-scale modeling of solute dispersion in unsaturated double-porosity media: homogenization and experimental validation
}

\author{
Tien Dung TRAN NGOC ${ }^{1, \star}$, Jolanta LEWANDOWSKA ${ }^{2, *}{ }^{*},, \S$, Michel VAUCLIN ${ }^{3}$ and Henri \\ BERTIN $^{4}$
}

${ }^{1} B R G M$ (French Geological Survey), Département Géothermie, 3 avenue Claude Guillemin, 45060 Orléans cedex 02, France.

${ }^{2}$ Laboratoire de Mécanique et Génie Civil (LMGC), UMR 5508 CNRS, Université Montpellier 2, CC 048 Place Eugène Bataillon, 34095 Montpellier cedex 5, France.

${ }^{3}$ Laboratoire d'étude des Transferts en Hydrologie et Environnement (LTHE), UMR 5564 CNRS, INPG, IRD, UJF, 38041 Grenoble cedex 09, France.

${ }^{4}$ Laboratoire TRansferts, Ecoulements, FLuides, Energétique (TREFLE), UMR 8508, CNRS, ENSAM, ENSCPB, Université de Bordeaux, 33405 Talence cedex, France.

\section{SUMMARY}

A two-scale modeling of solute transport in double-porosity media under unsaturated water flow conditions is presented. The macroscopic model was developed by applying the asymptotic homogenization method. It is based on theoretical and empirical considerations dealing with the orders of magnitude of characteristic quantities involved in the process. For this purpose a physical model which mimics the behaviour of double-porosity medium was built. The resulting two-equation model relies on a coupling exchange term between microand macro-porosity subdomains associated with local non-equilibrium solute concentrations. The model was numerically implemented (Comsol Multiphysics ${ }^{\circledR}$ ) to simulate the macroscopic one-dimensional physical process taking place into the porous medium of 3D periodic microstructure. A series of dispersion experiments of $\mathrm{NaCl}$ solution under unsaturated steady-state flow conditions were performed. The experimental results were used firstly to calibrate the dispersion coefficient of the model, and secondly to validate it through two other independent experiments. The excellent agreement between the numerical simulations and the measurements of the time evolution of the non-symmetrical breakthrough curves provides a proof of predictive capacity of the developed model.

KEYWORDS: unsaturated double-porosity media; homogenization; dispersion; local nonequilibrium transport; experiments; two-scale computations.

\footnotetext{
*Correspondence to: Jolanta.Lewandowska Laboratoire de Mécanique et Génie Civil (LMGC), UMR 5508 CNRS, Université Montpellier 2, CC 048 Place Eugène Bataillon, 34095 Montpellier cedex 5, France.

†E-mail: jolanta.lewandowska@univ-montp2.fr

*formerly at 3 and actually at Institut National de la Recherche Scientifique, INRS-Eau, Terre et Environnement, 490, rue de la Couronne, Québec, Québec, Canada.

${ }^{\S}$ formerly at Laboratoire Sols, Solides, Structures-Risques (3S-R), UMR 5521 UJF, CNRS, INPG, 38041

Grenoble cedex 09, France.
} 


\section{TWO-SCALE MODELING OF SOLUTE DISPERSION}

Submitted to International Journal for Numerical and Analytical Methods in Geomechanics 


\section{INTRODUCTION}

In the context of recent intensive development of new engineering fields such as deep storage of nuclear wastes, $\mathrm{CO}_{2}$ sequestration, waste landfills, or extraction of geothermal energy, large research efforts are directed toward efficient protection of subsurface water resources, which is of growing concern in public and regulatory agencies. Of special attention, is the prediction of water flow and solute transport in highly heterogeneous soils and rocks. A class of such media is modeled as double-porosity media. They are characterized by bi-modal pore size distribution, with micro- and macro-porosity. The double-porosity (DP) microstructure can arise as a consequence of different physical, mechanical, thermal or chemical processes. The typical examples are aggregated soils or fissured rocks. They show non-standard behaviour like preferential flow and transport that cannot be described by classical mathematical models.

Several models have been proposed for describing the preferential flow and transport in unsaturated double-porosity media. Most of them are phenomenological [1, 2, 3] and use the concept of two overlapping continua initially introduced by [4]. In these models, the DP medium is represented by two interacting domains, one associated with the soil aggregates or rock matrix blocks, and another one corresponding to the inter-aggregate pore space, macropores or fracture network. A number of such models exist in the literature and they differ by the assumptions concerning the mechanism of transport in both domains. Van Genuchten and Wierenga [5] proposed the mobile-immobile (MIM) model in which transport in the macroporosity domain is described by the convection-dispersion equation (CDE), while the transport in the micro-porosity domain is governed by the diffusion equation. The dualporosity model presented in [6], also named the dual-permeability mobile-mobile model (DPMM), employs the CDE approach in both domains. This model was extended to the triple-porosity medium to obtain the dual-permeability mobile-immobile (DPMIM) model [7] by assuming that water content in the matrix is partitioned into mobile and immobile fractions. Another concept was proposed in the MACRO model [8] which assumes that the solute transport is described by the CDE equation in the micro-porosity domain, while only convection is taken into account in the macro-porosity domain. In all these models, the solute exchange between the two domains is represented by a first-order term which is proportional to the difference in concentrations. Gerke and van Genuchten [9] discussed different possible expressions for the first-order solute mass transfer coefficient depending on structural soil geometry.

A number of experimental studies were carried out, from laboratory scale to field scale, to provide evidence of preferential flow and transport on one hand, and to verify the applicability of available models on the other hand. For example, solute short and large "loops" were observed in tracer in situ tests at the Soultz-Sous-Forêts deep geothermal site in France [10]. These experiments showed evidence of preferential hydraulic paths due to the fracture network [11]. By performing tracer displacement experiments in undisturbed soil column, Schwart et al. [12] pointed out the difficulty in estimating the parameters of the dualporosity model [6] by using an inverse technique, because of the non-linearity of the mass exchange term. Gerke and Köhne [13] succeeded in the validation of the DPMM model [6] through experimental observations of bromide transport in a tile-drained agricultural field by identifying the model parameters by inverse analysis, the geometrical transfer term being estimated empirically from the soil structure description. The MACRO model [8] was verified by two series of multiple tracers transport data in undisturbed soil column [14], one for the 
parameter estimation stage and another one for the validation stage. The comparison of the performance of different DP models: MIM [5], DPMM [6] and DPMIM [7] was assessed in Köhne et al. [15] by simulating experiments of herbicides transport in unsaturated intact soil column. The authors concluded that the DPMIM approach was the best way to reproduce the physical non-equilibrium transport. It must be noted that this model requires the largest number of parameters to be determined (see comparison of parameter number of different models in [16]). Although it was shown that the phenomenological DP models were able to reproduce the preferential transport observed in the experiments, some open questions were left remaining, especially concerning the origin of the macroscopic behaviour, the domain of validity of these models and the determination of the parameters. Moreover, the complex microstructure of natural media used in the experiments and the superposition of various biophysico-chemical processes made very difficult the distinction between different effects.

The phenomena of water flow and solute transport in DP media have also been modeled by using different micro-macro upscaling methods. In these approaches, macroscopic models are derived from the description of physical processes occurring at the microscopic scale. For example, a two-equation model [17] describing the solute transport in saturated heterogeneous medium was developed by employing the volume averaging technique [18]. In [19], this technique was applied to develop a large-scale dispersion model for the case of heterogeneous porous medium and the two-phase flow. Results showed that this kind of modeling is facing several limiting assumption, and the authors concluded that "even with the simplifying assumptions the large-scale dispersion coefficients exhibit very complex behaviour".

Alternatively, the asymptotic homogenization method was used to obtain different flow and transport models in double-porosity media. The homogenized models were reviewed by Peszynska and Showalter [20] for saturated conditions through a heterogeneous porous medium. Concerning more specifically the unsaturated DP media, Hornung [21] developed a macroscopic model of diffusion-convection coupled with a diffusion equation in the microporosity domain. Based on the Richard's equation [22], this model was associated with the DP approach describing unsaturated water flow. Using the same model for double-porosity unsaturated water flow, Mikelic and Rosier [23] proposed a transport model which takes into account convection and dispersion in the macro-porosity, coupled with adsorption at the micro-porosity external boundary. However, no solute transport in the micro-porosity was considered.

The aim of this paper is to present the macroscopic model of solute transport in unsaturated double-porosity medium obtained by asymptotic homogenization. This model is associated with the unsaturated water flow model which was previously developed by applying the homogenization method [27]. The solute transport model was validated through fully controlled $\mathrm{NaCl}$ dispersion experiments on a DP physical model of known microstructure, the same which was previously used for the validation of the double-porosity unsaturated water flow model $[24,25,26]$. The paper is structured as follows. Section 2 presents the assumptions and the formulation of the problem. The macroscopic model is developed in section 3. Section 4 aims at describing the tracer transport experiments in the physical model under steady-state unsaturated flow. In section 5, the validation of the theoretical model is performed by comparing experimental and numerical results. This section also gives the numerical implementation of the macroscopic model, using the Comsol Multiphysics ${ }^{\circledR}$ FEM code, for the case of $1 \mathrm{D}$ physical process taking place in a medium of 3D microstructure. The 
domain of validity of the approach is discussed in section 6 , and the main conclusions are summarized in section 7 .

\section{FORMULATION OF THE PROBLEM}

We consider solute transport in a double-porosity medium under unsaturated steady-state water flow conditions. In the following subsections, the general assumptions concerning the geometry, the microstructure of the medium and the formulation of the physical problems at the microscale, are presented. We also recall the main results of homogenization of the unsaturated water flow problem, published in [27]. Since low level of solute concentration was considered, the water flow problem can be decoupled from the solute transport problem in the sense that water flow velocity will be considered as an input parameter in the solute transport description.

\subsection{General assumptions}

We consider a rigid porous medium characterized by a period $(\Omega)$ and two porous subdomains $\left(\Omega_{1}\right.$ and $\left.\Omega_{2}\right)$ of contrasted physical properties and separated by a common interface $\Gamma$ (Figure 1). Since the porous medium presents a double structure, three different scales can be distinguished: the pore scale, the Darcy scale (named microscale or local scale) and the macroscopic scale, respectively. In this study, the starting point of the upscaling analysis is the Darcy scale. It is assumed that the porous sub-domain 1 (macro-porosity domain $\Omega_{1}$ ) is continuously connected and much more highly conductive than the porous sub-domain 2 (micro-porosity domain $\Omega_{2}$ ). Let us also assume the existence of scale separation expressed by a small parameter $\varepsilon$, defined by the ratio of two characteristic lengths: dimension of the period at the microscopic scale $\ell[\mathrm{L}]$ and dimension of the domain at the macroscopic scale $L[\mathrm{~L}]$. This condition is formally written as:

$$
\varepsilon=\frac{\ell}{L}<<1
$$

The condition (1) is equivalent to the existence of a representative elementary volume. The aim of the modelling is to study the asymptotic behaviour as $\varepsilon \rightarrow 0$. We denote $X=\left(X_{1}, X_{2}, X_{3}\right)$ [L] the physical spatial variable with $X_{3}$ oriented positively upward. In what follows the subscripts " 1 " and " 2 " denote the macro- and micro-porosity domain, respectively. By making use of the microscopic and macroscopic characteristic lengths, two dimensionless space variables are introduced:

$$
\boldsymbol{y}=\frac{\boldsymbol{X}}{\ell} \text { and } \boldsymbol{x}=\frac{\boldsymbol{X}}{L}
$$

where $\boldsymbol{y}\left(y_{1}, y_{2}, y_{3}\right)$ and $\boldsymbol{x}\left(x_{1}, x_{2}, x_{3}\right)$ are the microscopic and macroscopic dimensionless space variables respectively.

\subsection{Microscopic models of physical phenomena}

We consider the unsaturated water flow due to capillary and gravity forces under steady-state conditions with constant volumetric water contents in each subdomain, the air pressure being 
assumed constant and equal to the atmospheric pressure during the whole process. It is also assumed that solute is transported by convection and dispersion/diffusion without reaction, neither on the solid surface, nor in the fluid phase.

\section{Water flow formulation}

The water mass balance equations are written [22] as:

$\nabla_{X} \cdot\left[\boldsymbol{K}_{1}\left(h_{1}\right) \nabla_{X}\left(h_{1}+X_{3}\right)\right]=0$ in $\Omega_{1}$,

$\nabla_{X} \cdot\left[\boldsymbol{K}_{2}\left(h_{2}\right) \nabla_{X}\left(h_{2}+X_{3}\right)\right]=0$ in $\Omega_{2}$,

with the following continuity conditions at the interface:

$$
\begin{aligned}
& {\left[\boldsymbol{K}_{1}\left(h_{1}\right) \nabla_{X}\left(h_{1}+X_{3}\right)\right] \cdot \boldsymbol{N}=\left[\boldsymbol{K}_{2}\left(h_{2}\right) \nabla_{X}\left(h_{2}+X_{3}\right)\right] \cdot \boldsymbol{N} \text { on } \Gamma,} \\
& h_{1}\left(\theta_{1}\right)=h_{2}\left(\theta_{2}\right) \text { on } \Gamma,
\end{aligned}
$$

where $h(\theta)[\mathrm{L}]$ is the water capillary pressure head, $\theta\left[\mathrm{L}^{3} \mathrm{~L}^{-3}\right]$ is the volumetric water content, $\boldsymbol{K}\left[\mathrm{LT}^{-1}\right]$ is the unsaturated hydraulic conductivity tensor and $\boldsymbol{N}$ is the unit vector normal to $\Gamma$ and exterior to $\Omega_{1}$.

While the main results of homogenization of the problem (3)-(6) will be recalled in section 3, the focus is put hereafter on the modelling of the transport problem, associated with the unsaturated water flow.

\section{Solute transport formulation}

The solute transport is governed by the mass balance equations [28] as follows:

$$
\begin{aligned}
& \frac{\partial\left(\theta_{1} C_{1}\right)}{\partial t}=\nabla_{X} \cdot\left(\boldsymbol{D}_{1}\left(\theta_{1}\right) \nabla_{X} C_{1}-\boldsymbol{v}_{1} C_{1}\right) \text { in } \Omega_{1}, \\
& \frac{\partial\left(\theta_{2} C_{2}\right)}{\partial t}=\nabla_{X} \cdot\left(\boldsymbol{D}_{2}\left(\theta_{2}\right) \nabla_{X} C_{2}-\boldsymbol{v}_{2} C_{2}\right) \text { in } \Omega_{2},
\end{aligned}
$$

together with the continuity conditions of fluxes and concentrations at the interface:

$$
\begin{aligned}
& \left(\boldsymbol{D}_{1}\left(\theta_{1}\right) \nabla_{X} C_{1}-\boldsymbol{v}_{1} C_{1}\right) \cdot \boldsymbol{N}=\left(\boldsymbol{D}_{2}\left(\theta_{2}\right) \nabla_{X} C_{2}-\boldsymbol{v}_{2} C_{2}\right) \cdot \boldsymbol{N} \text { on } \Gamma, \\
& C_{1}=C_{2} \text { on } \Gamma,
\end{aligned}
$$

where $C\left[\mathrm{ML}^{-3}\right]$ is the solute concentration; $\boldsymbol{D}(\theta)\left[\mathrm{L}^{2} \mathrm{~T}^{-1}\right]$ is the local diffusion/dispersion tensor; $v\left[\mathrm{LT}^{-1}\right]$ is the local water flow velocity in the porous medium. The latter is assumed known and determined independently from the transport problem.

\subsection{Dimensionless variables}


In order to perform the homogenization process, we have to normalize the variables appearing in the transport problem (7)-(10) by introducing the characteristic values denoted by the subscript "c" [29]. It leads to the definition of a set of dimensionless variables:

$$
C_{1}^{*}=\frac{C_{1}}{C_{1 c}} ; C_{2}^{*}=\frac{C_{2}}{C_{2 c}} ; \boldsymbol{D}_{1}^{*}=\frac{\boldsymbol{D}_{1}}{D_{1 c}} ; \boldsymbol{D}_{2}^{*}=\frac{\boldsymbol{D}_{2}}{D_{2 c}} ; \boldsymbol{v}_{1}^{*}=\frac{\boldsymbol{v}_{1}}{v_{1 c}} ; \boldsymbol{v}_{2}^{*}=\frac{\boldsymbol{v}_{2}}{v_{2 c}} ; \theta_{1}^{*}=\frac{\theta_{1}}{\theta_{1 c}} ; \theta_{2}^{*}=\frac{\theta_{2}}{\theta_{2 c}} ; t^{*}=\frac{t}{t_{c}} \text {. }
$$

By using $\ell$ as the characteristic length, the local problem (7)-(10) is written for the dimensionless variables in the following form:

$$
\begin{aligned}
& \frac{\ell^{2} \theta_{1 c}}{D_{1 c} t_{c}} \frac{\partial\left(\theta_{1}^{*} C_{1}^{*}\right)}{\partial t^{*}}=\nabla_{y} \cdot\left(\boldsymbol{D}_{1}^{*} \nabla_{y} C_{1}^{*}-\frac{\ell v_{1 c}}{D_{1 c}} \boldsymbol{v}_{1}^{*} C_{1}^{*}\right) \text { in } \Omega_{1}, \\
& \frac{\ell^{2} \theta_{2 c}}{D_{2 c} t_{c}} \frac{\partial\left(\theta_{2}^{*} C_{2}^{*}\right)}{\partial t^{*}}=\nabla_{y} \cdot\left(\boldsymbol{D}_{2}^{*} \nabla_{y} C_{2}^{*}-\frac{\ell v_{2 c}}{D_{2 c}} \boldsymbol{v}_{2}^{*} C_{2}^{*}\right) \text { in } \Omega_{2}, \\
& \left(\boldsymbol{D}_{1}^{*} \nabla_{y} C_{1}^{*}-\frac{\ell v_{1 c}}{D_{1 c}} \boldsymbol{v}_{1}^{*} C_{1}^{*}\right) \cdot \boldsymbol{N}=\frac{D_{2 c}}{D_{1 c}} \frac{C_{2 c}}{C_{1 c}}\left(\boldsymbol{D}_{2}^{*} \nabla_{y} C_{2}^{*}-\frac{\ell v_{2 c}}{D_{2 c}} \boldsymbol{v}_{2}^{*} C_{2}^{*}\right) \cdot \boldsymbol{N} \text { on } \Gamma, \\
& C_{1 c} C_{1}^{*}=C_{2 c} C_{2}^{*} \text { on } \Gamma .
\end{aligned}
$$

From Equations (12) to (15) it can be seen that physical processes are governed by five dimensionless numbers:

- two Péclet numbers $P e$ describing the transport regime in each sub-domain:

$$
P e_{1 \ell}=\frac{\ell v_{1 c}}{D_{1 c}} \text { and } P e_{2 \ell}=\frac{\ell v_{2 c}}{D_{2 c}}
$$

- two numbers $P$ related to the characteristic time (time of the observation): $P_{1} \ell=$

$$
\frac{\ell^{2} \theta_{1 c}}{D_{1 c} t_{c}} \text { and } P_{2} \ell=\frac{\ell^{2} \theta_{2 c}}{D_{2 c} t_{c}}
$$

- a number $G$ corresponding to the ratio of the diffusion/dispersion coefficients in each sub-domain: $G=\frac{D_{2 c}}{D_{1 c}}$.

In the next section, the orders of magnitude of all the dimensionless numbers (16) will be evaluated using the powers of $\varepsilon$. This evaluation is based on experimental observations available in the litterature and some assumptions to be verified by the laboratory dispersion experiments using a physical model of double-porosity.

\subsection{Estimations of the orders of magnitude of the dimensionless numbers}

Let us recall that double-porosity media are characterized by the ratio of the hydraulic conductivities of the two sub-domains of the order $\boldsymbol{K}_{2} / \boldsymbol{K}_{1}=O\left(\varepsilon^{2}\right)$ [27]. It directly follows 
from the large contrast of the characteristic pore sizes in the macro- and micro-porosity. In the steady-state flow conditions, the water velocity in the micro-porosity disapears in the macroscopic flow model, since it was shown that water is trapped by capillary forces [27], $[24,25]$. Further, from several dispersion experiments published in the literature [30, 31, 32] we can learn that the characteristic time of the phenomenon corresponds to the transport by convection. Therefore, in this paper we take the characteristic time as equal to the time by convection through the macro-porosity domain

$$
t_{c}=\frac{L \theta_{1 c}}{v_{1 c}} .
$$

Then, two assumptions are made and that will be verified experimentally (section 6). The first assumption concerns the Péclet number $P e_{1} \ell$ which was assumed to be of the order one

$$
P e_{1 \ell}=\frac{\ell v_{1 c}}{D_{1 c}}=O(1) .
$$

As a consequence of this assumption we have $P_{1}=O(\varepsilon)$. The second assumption deals with the ratio $G$ assumed to be of the order $O\left(\varepsilon^{2}\right)$

$$
G=\frac{D_{2 c}}{D_{1 c}}=O\left(\varepsilon^{2}\right)
$$

Consequently, with the assumption of $\theta_{1 c}$ of the order of $\theta_{2 c}$, we have $P_{2 \ell}=O\left(\varepsilon^{-1}\right)$. An important consequence of these assumptions is that the local transport mechanism in the macro-porosity is convection and dispersion $\left(D_{1 \mathrm{c}}\right.$ is the characteristic value of the local dispersion coefficient) [33], while in the micro-porosity it is diffusion $\left(D_{2 \mathrm{c}}\right.$ is the characteristic value of the local diffusion coefficient). Since water is immobile in the micro-porosity, we have $P e_{2} e=0$. Finally, we assume $C_{1 c}$ to be of the same order of magnitude as $C_{2 c}$ at the interface $\Gamma$.

\subsection{Non-dimensional transport problem at the microscopic scale}

By means of the above estimations, the local problem (12) - (15) can be reformulated as follows :

$$
\begin{aligned}
& \varepsilon \frac{\partial\left(\theta_{1}^{*} C_{1}^{*}\right)}{\partial t^{*}}=\nabla_{y} \cdot\left(\boldsymbol{D}_{1}^{*} \nabla_{y} C_{1}^{*}-\boldsymbol{v}_{1}^{*} C_{1}^{*}\right) \text { in } \Omega_{1}, \\
& \varepsilon^{-1} \frac{\partial\left(\theta_{2}^{*} C_{2}^{*}\right)}{\partial t^{*}}=\nabla_{y} \cdot\left(\boldsymbol{D}_{2}^{*} \nabla_{y} C_{2}^{*}\right) \text { in } \Omega_{2}, \\
& \left(\boldsymbol{D}_{1}^{*} \nabla_{y} C_{1}^{*}-\boldsymbol{v}_{1}^{*} C_{1}^{*}\right) \cdot \boldsymbol{N}=\varepsilon^{2}\left(\boldsymbol{D}_{2}^{*} \nabla_{y} C_{2}^{*}\right) \cdot \boldsymbol{N} \text { on } \Gamma, \\
& C_{1}^{*}=C_{2}^{*} \text { on } \Gamma .
\end{aligned}
$$


We can notice the presence of powers of $\varepsilon$ in front of some terms in Equations (20)-(22) which comes from the physical considerations. In section 3, the problem (20)-(23) will be homogenized to derive the macroscopic model by using the asymptotic homogenization method [34, 35] and [29]. We start by recalling the main results of homogenization of water flow problem that were given in a previous paper [27]. Some modifications were made in order to take into account the steady-state case considered in this paper.

\section{HOMOGENIZATION}

The homogenization process postulates that all unknowns $\psi$ can be expressed in the form of two-scale asymptotic developments, using the small parameter $\varepsilon$ [34, 35]:

$\psi\left(\boldsymbol{x}, \boldsymbol{y}, t^{*}\right)=\psi^{(0)}\left(\boldsymbol{x}, \boldsymbol{y}, t^{*}\right)+\varepsilon \psi^{(1)}\left(\boldsymbol{x}, \boldsymbol{y}, t^{*}\right)+\varepsilon^{2} \psi^{(2)}\left(\boldsymbol{x}, \boldsymbol{y}, t^{*}\right)+\ldots$

where all $\psi^{(i)}\left(\boldsymbol{x}, \boldsymbol{y}, t^{*}\right)$ are spatially periodic with respect to the variable $\boldsymbol{y}$. Due to (2) we have the relation $\boldsymbol{x}=\varepsilon \boldsymbol{y}$ that makes the derivation operator be transformed into

$\nabla_{y} \nabla \nabla t y+\varepsilon \nabla_{x}$

The homogenization process is classical and follows the methodology proposed by Auriault [29].

\subsection{Homogenization of the problem of water flow in unsaturated double porosity media}

The homogenization of the water flow problem, Equations (3)-(6), was presented in details in [27]. Note that this problem is highly non linear. The obtained results will be used in the homogenization of the transport problem.

The solution of the problem (20)-(21) given in [27] provided the macroscopic variable which is independent of the local variable $y$

$$
h_{1}^{(0)}=h_{1}^{(0)}\left(\boldsymbol{x}, t^{*}\right)=h^{(0)}\left(\boldsymbol{x}, t^{*}\right),
$$

where $h_{1}^{(0)}$ is the macroscopic capillary pressure head.

The next order problem (24)-(25)-(27) developed in [27] allowed to obtain the solution for $h_{1}^{(1)}$ in the form of

$$
h_{1}^{(1)}=\boldsymbol{\xi}(\boldsymbol{y})\left(\nabla_{x} h_{1}^{(0)}+e_{3}\right)+\bar{h}_{1}^{(1)}\left(\boldsymbol{x}, t^{*}\right)
$$

where $\boldsymbol{\xi}(\boldsymbol{y})$ is the characteristic vectorial function and $\bar{h}_{1}^{(1)}\left(\boldsymbol{x}, t^{*}\right)$ is an arbitrary function.

Finally, from (30)-(31) given in [27] and for the steady-state regime established in a DP medium, the macroscopic equation reads as:

$\nabla_{x} \cdot\left[\boldsymbol{K}^{\mathrm{eff}}\left(h^{(0)}\right) \nabla_{x}\left(h^{(0)}+x_{3}\right)\right]=0$, 
where

$$
\left\langle\boldsymbol{v}^{(0)}\right\rangle=\left\langle\boldsymbol{v}_{1}^{(0)}\right\rangle=\boldsymbol{K}^{\mathrm{eff}}\left(h^{(0)}\right) \nabla_{x}\left(h^{(0)}+x_{3}\right)
$$

is the macroscopic water flow velocity $\left[\mathrm{LT}^{-1}\right]$ and $\boldsymbol{K}^{\mathrm{eff}}\left[\mathrm{LT}^{-1}\right]$ is the effective hydraulic conductivity tensor of the double-porosity medium, defined by

$$
\boldsymbol{K}^{\mathrm{eff}}\left(h^{(0)}\right)=\frac{1}{|\Omega|} \int_{\Omega_{1}} \boldsymbol{K}_{1}^{(0)}\left(\nabla_{y} \boldsymbol{\xi}+\boldsymbol{I}\right) d \Omega
$$

where $\boldsymbol{I}$ is the identity matrix.

\subsection{Homogenization of the problem of solute transport in unsaturated double-porosity media}

This section presents the homogenization process of the transport problem, Equations (20)(23). Introducing the asymptotic developpments (24), taking into account (25), and regrouping the terms at the same powers of $\varepsilon$, lead to:

$$
\begin{aligned}
& \varepsilon \frac{\partial\left(\theta_{1}^{*} C_{1}^{(0)}\right)}{\partial t^{*}}+\varepsilon^{2} \frac{\partial\left(\theta_{1}^{*} C_{1}^{(1)}\right)}{\partial t^{*}}+\ldots= \\
& \nabla_{y} \cdot\left[\boldsymbol{D}_{1}^{*} \nabla_{y} C_{1}^{(0)}-\boldsymbol{v}_{1}^{(0)} C_{1}^{(0)}\right]+ \\
&+ \varepsilon\left[\nabla_{y} \cdot\left(\boldsymbol{D}_{1}^{*} \nabla_{y} C_{1}^{(1)}+\boldsymbol{D}_{1}^{*} \nabla_{x} C_{1}^{(0)}\right)-\nabla_{y} \cdot\left(\boldsymbol{v}_{1}^{(0)} C_{1}^{(1)}+\boldsymbol{v}_{1}^{(1)} C_{1}^{(0)}\right)-\nabla_{x} \cdot\left(\boldsymbol{D}_{1}^{*} \nabla_{y} C_{1}^{(0)}\right)-\nabla_{x} \cdot\left(\boldsymbol{v}_{1}^{(0)} C_{1}^{(0)}\right)\right]+ \\
&+ \varepsilon^{2}\left[\nabla_{y} \cdot\left(\boldsymbol{D}_{1}^{*} \nabla_{y} C_{1}^{(2)}+\boldsymbol{D}_{1}^{*} \nabla_{x} C_{1}^{(1)}\right)-\nabla_{y} \cdot\left(\boldsymbol{v}_{1}^{(0)} C_{1}^{(2)}+\boldsymbol{v}_{1}^{(1)} C_{1}^{(1)}+\boldsymbol{v}_{1}^{(2)} C_{1}^{(0)}\right)+\nabla_{x} \cdot \boldsymbol{D}_{1}^{*}\left(\nabla_{y} C_{1}^{(1)}+\nabla_{x} C_{1}^{(0)}\right)-\right]+\ldots \\
&\left.-\nabla_{x} \cdot\left(\boldsymbol{v}_{1}^{(0)} C_{1}^{(1)}+\boldsymbol{v}_{1}^{(1)} C_{1}^{(0)}\right)\right]
\end{aligned}
$$

in $\Omega_{1}$,

$$
\begin{aligned}
& \varepsilon^{-1} \frac{\partial\left(\theta_{2}^{*} C_{2}^{(0)}\right)}{\partial t^{*}}+\varepsilon \frac{0}{0} \frac{\partial\left(\theta_{2}^{*} C_{2}^{(1)}\right)}{\partial t^{*}}+\varepsilon \frac{\partial\left(\theta_{2}^{*} C_{2}^{(2)}\right)}{\partial t^{*}}+\ldots= \\
& \nabla_{y} \cdot\left(\boldsymbol{D}_{2}^{*} \nabla_{y} C_{2}^{(0)}\right)+\varepsilon^{1}\left[\nabla_{y} \cdot\left(\boldsymbol{D}_{2}^{*} \nabla_{y} C_{2}^{(1)}\right)+\nabla_{y} \cdot\left(\boldsymbol{D}_{2}^{*} \nabla_{x} C_{2}^{(0)}\right)+\nabla_{x} \cdot\left(\boldsymbol{D}_{2}^{*} \nabla_{y} C_{2}^{(0)}\right)\right]+\ldots
\end{aligned}
$$

$$
\begin{aligned}
& \left(\boldsymbol{D}_{1}^{*} \nabla_{y} C_{1}^{(0)}-\boldsymbol{v}_{1}^{(0)} C_{1}^{(0)}\right) \cdot \boldsymbol{N} \\
& +\varepsilon\left[\left(\boldsymbol{D}_{1}^{*} \nabla_{y} C_{1}^{(1)}+\boldsymbol{D}_{1}^{*} \nabla_{y} C_{1}^{(0)}-\boldsymbol{v}_{1}^{(0)} C_{1}^{(1)}-\boldsymbol{v}_{1}^{(1)} C_{1}^{(0)}\right) \cdot \boldsymbol{N}\right]+ \\
& +\varepsilon^{2}\left[\left(\boldsymbol{D}_{1}^{*} \nabla_{y} C_{1}^{(2)}+\boldsymbol{D}_{1}^{*} \nabla_{x} C_{1}^{(0)}-\boldsymbol{v}_{1}^{(0)} C_{1}^{(2)}-\boldsymbol{v}_{1}^{(1)} C_{1}^{(1)}-\boldsymbol{v}_{1}^{(2)} C_{1}^{(0)}\right) \cdot \boldsymbol{N}\right]+\ldots=\varepsilon^{2}\left(\boldsymbol{D}_{2}^{*} \nabla_{y} C_{2}^{(0)}\right) \cdot \boldsymbol{N}+\ldots \\
& \text { on } \Gamma, \\
& \left(C_{1}^{(0)}+\varepsilon C_{1}^{(1)}+\varepsilon^{2} C_{1}^{(2)}+\ldots\right)=\left(C_{2}^{(0)}+\varepsilon C_{2}^{(1)}+\varepsilon^{2} C_{2}^{(2)}+\ldots\right) \text { on } \Gamma
\end{aligned}
$$


Equating the terms of the same order of $\varepsilon$ in (31)-(34) yields successive boundary value problems to be solved within the period domain. In the following, these problems and their solutions are addressed.

\section{Macroscopic variable}

At the order $\varepsilon^{0}$, Equations (31) and (33) are written as:

$$
\begin{aligned}
& \nabla_{y} \cdot\left(\boldsymbol{D}_{1}^{*} \nabla_{y} C_{1}^{(0)}-\boldsymbol{v}_{1}^{(0)} C_{1}^{(0)}\right)=0 \text { in } \Omega_{1}, \\
& \left(\boldsymbol{D}_{1}^{*} \nabla_{y} C_{1}^{(0)}-\boldsymbol{v}_{1}^{(0)} C_{1}^{(0)}\right) \cdot \boldsymbol{N}=0 \text { on } \Gamma,
\end{aligned}
$$

where $C_{1}^{(0)}$ is $\boldsymbol{y}$-periodic. It can be shown that the solution of the problem (35) and (36) is

$$
C_{1}^{(0)}=C_{1}^{(0)}\left(\boldsymbol{x}, t^{*}\right)=C^{(0)}\left(\boldsymbol{x}, t^{*}\right) .
$$

It means that $C_{1}^{(0)}$ is the macroscopic variable, not depending on the local space variable $y$ and it is denoted $C^{(0)}$.

\section{Determination of $C_{1}^{(1)}$ and the local boundary value problem}

Let us analyse Equation (31) at the order $O\left(\varepsilon^{1}\right)$. Taking into account the solution (37) we obtain

$$
\frac{\partial\left(\theta_{1}^{*} C_{1}^{(0)}\right)}{\partial t^{*}}=\nabla_{y} \cdot\left[\boldsymbol{D}_{1}^{*}\left(\nabla_{y} C_{1}^{(1)}+\nabla_{x} C_{1}^{(0)}\right)-\left(\boldsymbol{v}_{1}^{(0)} C_{1}^{(1)}+\boldsymbol{v}_{1}^{(1)} C_{1}^{(0)}\right)\right]-\nabla_{x} \cdot\left(\boldsymbol{v}_{1}^{(0)} C_{1}^{(0)}\right) \text { in } \Omega_{1} .
$$

From the interface condition (33), we get at the order $O\left(\varepsilon^{1}\right)$ :

$$
\left(\boldsymbol{D}_{1}^{*} \nabla_{y} C_{1}^{(1)}+\boldsymbol{D}_{1}^{*} \nabla_{x} C_{1}^{(0)}-\boldsymbol{v}_{1}^{(0)} C_{1}^{(1)}-\boldsymbol{v}_{1}^{(1)} C_{1}^{(0)}\right) \cdot \boldsymbol{N}=0 \text { on } \Gamma \text {. }
$$

Firstly, we integrate Equation (38) over the domain $\Omega_{1}$, and divide it by $|\Omega|$. Then, we perform three transformations of the first term in the r.h.s. as follows: (i) apply the Gauss-Ostrogradski theorem to transform the volume integral to the surface integral; (ii) apply the periodicity condition and the boundary condition (39); (iii) apply once again the Gauss-Ostrogradski theorem to pass from the surface to the volume integral over the domain $\Omega_{2}$. We finally obtain

$$
\frac{\partial\left\langle\theta_{1}^{*} C_{1}^{(0)}\right\rangle}{\partial t^{*}}=-\nabla_{x} \cdot\left(\left\langle v_{1}^{(0)}\right\rangle C_{1}^{(0)}\right)
$$

Note that the volume average in (40) is defined by

$$
\langle\cdot\rangle=\frac{1}{|\Omega|} \int_{\Omega_{1}} \cdot d \Omega \text {. }
$$




\section{TWO-SCALE MODELING OF SOLUTE DISPERSION}

Because of (37) and the fact that $\theta_{1}{ }^{*}$ is $\boldsymbol{y}$-independent we can write

$$
\frac{\partial\left(\theta_{1}^{*} C_{1}^{(0)}\right)}{\partial t^{*}}=-\frac{1}{\phi_{1}} \nabla_{x} \cdot\left(\left\langle\boldsymbol{v}_{1}^{(0)}\right\rangle C_{1}^{(0)}\right)
$$

where $\phi_{1}=\left|\Omega_{1}\right| /|\Omega|$.

From (42), it can be seen that the convective behaviour of $C^{(0)}$ is obtained at the first order of approximation.

Now, introducing Equation (42) back into Equation (38) leads to:

$\nabla_{y} \cdot\left[\boldsymbol{D}_{1}^{*}\left(\nabla_{y} C_{1}^{(1)}+\nabla_{x} C_{1}^{(0)}\right)\right]-\nabla_{y} \cdot\left(\boldsymbol{v}_{1}^{(0)} C_{1}^{(1)}+\boldsymbol{v}_{1}^{(1)} C_{1}^{(0)}\right)-\nabla_{x} \cdot\left(\boldsymbol{v}_{1}^{(0)} C_{1}^{(0)}\right)=-\frac{1}{\phi_{1}} \nabla_{x} \cdot\left(\left\langle\boldsymbol{v}_{1}^{(0)}\right\rangle C_{1}^{(0)}\right)$

in $\Omega_{1}$,

The problem (43) and (39) defines $C_{1}^{(1)}$. Note that it is the same problem as obtained in [36] for the case of dispersion in a saturated simple-porosity (SP) medium. The solution of this problem can be put in the form

$$
C_{1}^{(1)}=\chi(\boldsymbol{y}) \nabla_{x} C_{1}^{(0)}+\bar{C}_{1}^{(1)}\left(\boldsymbol{x}, t^{*}\right),
$$

where the periodic vectorial function $\chi(y)$ characterizes the microstructure of the medium and $\bar{C}_{1}^{(1)}\left(\boldsymbol{x}, t^{*}\right)$ is an arbitrary function.

In order to obtain the local boundary value problem allowing to calculate $\chi(y)$, we replace (44) in (43), and we analyze the terms in the 1.h.s. Beginning by the first term, we obtain

$$
\nabla_{y} \cdot\left(\boldsymbol{D}_{1}^{*} \nabla_{y} C_{1}^{(1)}+\boldsymbol{D}_{1}^{*} \nabla_{x} C_{1}^{(0)}\right)=\nabla_{y} \cdot\left[\boldsymbol{D}_{1}^{*}\left(\nabla_{y} \boldsymbol{\chi}+\boldsymbol{I}\right)\right] \nabla_{x} C_{1}^{(0)} .
$$

The second term can be written

$$
\nabla_{y} \cdot\left(\boldsymbol{v}_{1}^{(0)} C_{1}^{(1)}+\boldsymbol{v}_{1}^{(1)} C_{1}^{(0)}\right)=C_{1}^{(1)} \nabla_{y} \cdot \boldsymbol{v}_{1}^{(0)}+\boldsymbol{v}_{1}^{(0)} \nabla_{y} C_{1}^{(1)}+C_{1}^{(0)} \nabla_{y} \cdot \boldsymbol{v}_{1}^{(1)}+\boldsymbol{v}_{1}^{(1)} \nabla_{y} C_{1}^{(0)} .
$$

Since we have $\nabla_{y} \cdot \boldsymbol{v}_{1}^{(0)}=0$ (Equation (28) in [27]) and $C_{1}^{(0)}$ does not depend on $\boldsymbol{y}$ (Equation (37) in the present paper), Equation (46) becomes

$$
\nabla_{y} \cdot\left(\boldsymbol{v}_{1}^{(0)} C_{1}^{(1)}+\boldsymbol{v}_{1}^{(1)} C_{1}^{(0)}\right)=\boldsymbol{v}_{1}^{(0)} \nabla_{y} \cdot\left(\chi \nabla_{x} C_{1}^{(0)}\right)+C_{1}^{(0)} \nabla_{y} \cdot \boldsymbol{v}_{1}^{(1)}
$$

The third term in (43) is

$$
\nabla_{x} \cdot\left(\boldsymbol{v}_{1}^{(0)} C_{1}^{(0)}\right)=C_{1}^{(0)} \nabla_{x} \cdot \boldsymbol{v}_{1}^{(0)}+\boldsymbol{v}_{1}^{(0)} \nabla_{x} C_{1}^{(0)}
$$

Finally, using Equations (45)-(48), Equation (43) is written in the following form 


$$
\begin{aligned}
& \left.\nabla_{y} \cdot \mid \boldsymbol{D}_{1}^{*}\left(\nabla_{y} \boldsymbol{\chi}+\boldsymbol{I}\right) \nabla_{x} C_{1}^{(0)}\right]-\boldsymbol{v}_{1}^{(0)} \nabla_{y} \cdot\left(\chi \nabla_{x} C_{1}^{(0)}\right)-C_{1}^{(0)}\left(\nabla_{y} \cdot \boldsymbol{v}_{1}^{(1)}+\nabla_{x} \cdot \boldsymbol{v}_{1}^{(0)}\right)= \\
& -\frac{1}{\phi_{1}}\left(C_{1}^{(0)} \nabla_{x} \cdot\left\langle\boldsymbol{v}_{1}^{(0)}\right\rangle+\left\langle\boldsymbol{v}_{1}^{(0)}\right\rangle \nabla_{x} C_{1}^{(0)}\right)+\boldsymbol{v}_{1}^{(0)} \nabla_{x} C_{1}^{(0)}
\end{aligned}
$$

The third term in the 1.h.s. of Equation (49) vanishes because of Equation (32) in [27] in the steady-state condition, and the first term in the brackets in the r.h.s. vanishes because of (28). Hence, Equation (49) is written as follows:

$$
\nabla_{y} \cdot\left[\boldsymbol{D}_{1}^{*}\left(\nabla_{y} \boldsymbol{\chi}+\boldsymbol{I}\right) \nabla_{x} C_{1}^{(0)}\right]-\boldsymbol{v}_{1}^{(0)} \nabla_{y} \cdot\left(\boldsymbol{\chi} \nabla_{x} C_{1}^{(0)}\right)=\left(\boldsymbol{v}_{1}^{(0)}-\frac{1}{\phi_{1}}\left\langle\boldsymbol{v}_{1}^{(0)}\right\rangle\right) \nabla_{x} C_{1}^{(0)}
$$

Imposing succesively the unit macroscopic gradient $\nabla_{x} C_{1}^{(0)}$ in Equation (50) for the directions 1,2 and 3 , we obtain the local problem for $\chi(y)$

$$
\nabla_{y} \cdot\left[D_{1}^{*}\left(\nabla_{y} \chi+I\right)\right]-v_{1}^{(0)} \nabla_{y} \chi=v_{1}^{(0)}-\frac{1}{\phi_{1}}\left\langle\boldsymbol{v}_{1}^{(0)}\right\rangle
$$

Taking into account Equations (25) and (33) in [27] for the conditions of steady-state water flow, as well as the solution obtained for $C_{1}^{(1)}$ (Equation (44)), Equation (39) is re-written

$$
\left\lfloor\boldsymbol{D}_{1}^{*}\left(\nabla_{y} \chi+\boldsymbol{I}\right) \mid \cdot \boldsymbol{N}=0 \text { on } \Gamma\right.
$$

where $\chi$ in Equation (51) - (52) is y-periodic with the following additional condition:

$$
\langle\chi\rangle=\frac{1}{|\Omega|} \int_{\Omega_{1}} \chi d \Omega=0
$$

The local boundary value problem $(51)-(53)$ defines the characteristic function $\chi$. This local boundary value problem possesses the same form as that obtained by Auriault and Adler [37], and Auriault and Lewandowska [36] for dispersion in saturated conditions in a SP medium. Here, $\boldsymbol{D}_{1}^{*}$ is the local dispersion tensor which depends on the local velocity.

\subsection{Macroscopic model}

The macroscopic model is obtained by analyzing the problem (31) and (33) at the order $O\left(\varepsilon^{2}\right)$

$$
\begin{array}{r}
\frac{\partial\left(\theta_{1}^{*} C_{1}^{(1)}\right)}{\partial t^{*}}=\nabla_{y} \cdot\left[\boldsymbol{D}_{1}^{*}\left(\nabla_{y} C_{1}^{(2)}+\nabla_{x} C_{1}^{(1)}\right)\right]+\nabla_{x} \cdot\left[\boldsymbol{D}_{1}^{*}\left(\nabla_{y} C_{1}^{(1)}+\nabla_{x} C_{1}^{(0)}\right)\right]-\nabla_{y} \cdot\left(\boldsymbol{v}_{1}^{(0)} C_{1}^{(2)}\right)- \\
-\nabla_{y} \cdot\left(\boldsymbol{v}_{1}^{(1)} C_{1}^{(1)}\right)-\nabla_{y} \cdot\left(\boldsymbol{v}_{1}^{(2)} C_{1}^{(0)}\right)-\nabla_{x} \cdot\left(\boldsymbol{v}_{1}^{(0)} C_{1}^{(1)}\right)-\nabla_{x} \cdot\left(\boldsymbol{v}_{1}^{(1)} C_{1}^{(0)}\right)
\end{array}
$$

in $\Omega_{1}$,

$$
\left(\boldsymbol{D}_{1}^{*} \nabla_{y} C_{1}^{(2)}+\boldsymbol{D}_{1}^{*} \nabla_{x} C_{1}^{(1)}-\boldsymbol{v}_{1}^{(0)} C_{1}^{(2)}-\boldsymbol{v}_{1}^{(1)} C_{1}^{(1)}-\boldsymbol{v}_{1}^{(2)} C_{1}^{(0)}\right) \cdot \boldsymbol{N}=\left(\boldsymbol{D}_{2}^{*} \nabla_{y} C_{2}^{(0)}\right) \cdot \boldsymbol{N} \text { on } \Gamma \text {. }
$$


Concerning the transport problem in the micro-porosity domain, from Equation (32) we obtain at successive orders the following equations:

$\frac{\partial\left(\theta_{2}^{*} C_{2}^{(0)}\right)}{\partial t^{*}}=0$ in $\Omega_{2}$

$\frac{\partial\left(\theta_{2}^{*} C_{2}^{(1)}\right)}{\partial t^{*}}=\nabla_{y} \cdot\left(\boldsymbol{D}_{2}^{*} \nabla_{y} C_{2}^{(0)}\right)$ in $\Omega_{2}$.

Now, let us postulate that Equation (23) is replaced by the following condition for the concentration on the interface $\Gamma$

$\frac{1}{\left|\Omega_{1}\right|} \int_{\Omega_{1}} C_{1}^{*} d \Omega=C_{2}^{*}$ or $\left\langle C_{1}^{*}\right\rangle_{\Omega_{1}}=C_{2}^{*}$ on $\Gamma$

which means

$\left.\left.\left(<C_{1}^{(0)}\right\rangle_{\Omega_{1}}+\varepsilon<C_{1}^{(1)}\right\rangle_{\Omega_{1}}+\varepsilon^{2}\left\langle C_{1}^{(2)}\right\rangle_{\Omega_{1}}+\ldots\right)=\left(C_{2}^{(0)}+\varepsilon C_{2}^{(1)}+\varepsilon^{2} C_{2}^{(2)}+\ldots\right)$ on $\Gamma$

and thus

$\left.<C_{1}^{(0)}\right\rangle_{\Omega_{1}}=C_{2}^{(0)}$ and $\left\langle C_{1}^{(1)}\right\rangle_{\Omega_{1}}=C_{2}^{(1)}$ on $\Gamma$.

From (56), it can be concluded that $C_{2}^{(0)}$ is time-independent, but can depend on $(\boldsymbol{x}, \boldsymbol{y})$. This conclusion is in contradiction with the solution for $C_{1}^{(0)}$ as time-dependent, Equation (37). Therefore, it would require the introduction of a boundary layer in order to match the interface condition. On the contrary, $C_{2}^{(1)}$, the solution of (57), is time-dependent and gives rise to the mass exchange between the micro- and macro-porosity. In this paper, the verification of the global interface condition (58) will be postulated only.

In order to get the macroscopic description, Equation (54) is integrated over the domain $\Omega_{1}$, and divided by $|\Omega|$. Then, several transformations are performed to get

$\left.\left.\frac{\partial\left\langle\theta_{1}^{*} C_{1}^{(1)}\right\rangle}{\partial t^{*}}=\nabla_{x} \cdot\left\langle\boldsymbol{D}_{1}^{*}\right| \nabla_{y} C_{1}^{(1)}+\nabla_{x} C_{1}^{(0)}\right)\right\rangle-\nabla_{x} \cdot\left\langle\boldsymbol{v}_{1}^{(0)} C_{1}^{(1)}\right\rangle-\nabla_{x} \cdot\left\langle\boldsymbol{v}_{1}^{(1)} C_{1}^{(0)}\right\rangle-\frac{\partial\left\langle\theta_{2}^{*} C_{2}^{(1)}\right\rangle}{\partial t^{*}}$.

By substituting (44) into (61), we obtain

$\frac{\partial\left\langle\theta_{1}^{*} C_{1}^{(1)}\right\rangle}{\partial t^{*}}=\nabla_{x} \cdot\left(\boldsymbol{D}_{\text {disp }}^{*} \nabla_{x} C_{1}^{(0)}\right)-\nabla_{x} \cdot\left(\left\langle\boldsymbol{v}_{1}^{(0)}\right\rangle \bar{C}_{1}^{(1)}\right)-\nabla_{x} \cdot\left(\left\langle\boldsymbol{v}_{1}^{(1)}\right\rangle C_{1}^{(0)}\right)-\frac{\partial\left\langle\theta_{2}^{*} C_{2}^{(1)}\right\rangle}{\partial t^{*}}(62)$ 
where

$$
\boldsymbol{D}_{\mathrm{disp}}^{*}=\left\langle\boldsymbol{D}_{1}^{*}\left[\left(\nabla_{y} \boldsymbol{\chi}+I\right)-\boldsymbol{v}_{1}^{(0)} \boldsymbol{\chi}\right]\right\rangle=\frac{1}{|\Omega|} \int_{\Omega_{1}} \boldsymbol{D}_{1}^{*}\left[\left(\nabla_{y} \boldsymbol{\chi}+\boldsymbol{I}\right)-\boldsymbol{v}_{1}^{(0)} \boldsymbol{\chi}\right] d \Omega
$$

is the dispersion tensor of the DP medium. Note that its definition is the same as in the case of saturated SP medium ([37] and [36]).

Then, Equation (40) and Equation (Erreur : source de la référence non trouvée) multiplied by $\varepsilon$, are added leading to

$$
\begin{array}{r}
\frac{\partial\left\langle\theta_{1}^{*} C_{1}^{(0)}\right\rangle}{\partial t^{*}}+\varepsilon \frac{\partial\left\langle\theta_{1}^{*} C_{1}^{(1)}\right\rangle}{\partial t^{*}}=\varepsilon \nabla_{x} \cdot\left(\boldsymbol{D}_{\mathrm{disp}}^{*} \nabla_{x} C_{1}^{(0)}\right)-\varepsilon \nabla_{x} \cdot\left\langle\boldsymbol{v}_{1}^{(0)} \bar{C}_{1}^{(1)}\right\rangle-\varepsilon \nabla_{x} \cdot\left\langle\boldsymbol{v}_{1}^{(1)} C_{1}^{(0)}\right\rangle- \\
-\nabla_{x} \cdot\left(\left\langle\boldsymbol{v}_{1}^{(0)}\right\rangle C_{1}^{(0)}\right)-\varepsilon \frac{\partial\left\langle\theta_{2}^{*} C_{2}^{(1)}\right\rangle}{\partial t^{*}}
\end{array}
$$

In the following, we make use of the expressions:

$$
\begin{aligned}
& \left\langle\boldsymbol{v}_{1}^{*}\right\rangle=\left\langle\boldsymbol{v}_{1}^{(0)}\right\rangle+\varepsilon\left\langle\boldsymbol{v}_{1}^{(1)}\right\rangle+\ldots \\
& \left\langle C_{1}^{*}\right\rangle=\left\langle C_{1}^{(0)}\right\rangle+\varepsilon\left\langle C_{1}^{(1)}\right\rangle+\ldots \approx \phi_{1} C_{1}^{(0)}+\phi_{1} \varepsilon \bar{C}_{1}^{(1)} \text { and }\left\langle C_{2}^{*}\right\rangle=\left\langle C_{2}^{(0)}\right\rangle+\varepsilon\left\langle C_{2}^{(1)}\right\rangle+\ldots
\end{aligned}
$$

where

$$
\left\langle C_{1}^{*}\right\rangle=\frac{1}{|\Omega|} \int_{\Omega_{1}} C_{1}^{*} d \Omega \quad \text { and }\left\langle C_{2}^{*}\right\rangle=\frac{1}{|\Omega|} \int_{\Omega_{2}} C_{2}^{*} d \Omega
$$

It allows us to put together the three convective terms in the r.h.s. of Equation (Erreur : source de la référence non trouvée) in the form of

$$
\varepsilon \nabla_{x} \cdot\left(\left\langle v_{1}^{(0)} \bar{C}_{1}^{(1)}\right\rangle\right)+\varepsilon \nabla_{x} \cdot\left(\left\langle\boldsymbol{v}_{1}^{(1)} C_{1}^{(0)}\right\rangle\right)+\nabla_{x} \cdot\left(\left\langle\boldsymbol{v}_{1}^{(0)}\right\rangle C_{1}^{(0)}\right)=\frac{1}{\phi_{1}} \nabla_{x} \cdot\left(\left\langle\boldsymbol{v}_{1}^{*}\right\rangle\left\langle C_{1}^{*}\right\rangle\right)+O\left(\varepsilon^{2}\right) .
$$

Taking into account (68), we can write the Equation (Erreur : source de la référence non trouvée) in the form

$$
\frac{\partial\left\langle\theta_{1}^{*} C_{1}^{0}\right\rangle}{\partial t^{*}}+\varepsilon \frac{\partial\left\langle\theta_{1}^{*} C_{1}^{1}\right\rangle}{\partial t^{*}}=\frac{1}{\phi_{1}} \varepsilon \nabla_{x} \cdot\left(\boldsymbol{D}_{\mathrm{disp}}^{*} \nabla_{x}\left\langle C_{1}^{(0)}\right\rangle\right)-\frac{1}{\phi_{1}} \nabla_{x} \cdot\left(\left\langle\boldsymbol{v}_{1}^{*}\right\rangle\left\langle C_{1}^{*}\right\rangle\right)-\varepsilon \frac{\partial\left\langle\theta_{2}^{*} C_{2}^{1}\right\rangle}{\partial t^{*}} .
$$

Finally, by considering that $\theta_{1}$ and $\theta_{2}$ are $\boldsymbol{y}$-independent, and taking into account (66) and (56), Equation (69) can be written for the average $\left\langle C_{1}^{*}\right\rangle_{\Omega_{1}}$ with the precision $O\left(\varepsilon^{2}\right)$ as: 
$\phi_{1} \frac{\left.\partial\left(\theta_{1}^{*}<C_{1}^{*}\right\rangle_{\Omega_{1}}\right)}{\partial t^{*}}=\varepsilon \nabla_{x} \cdot\left(\boldsymbol{D}_{\text {disp }}^{*} \nabla_{x}<C_{1}^{*}>_{\Omega_{1}}\right)-\nabla_{x} \cdot\left(\left\langle\boldsymbol{v}_{1}^{*}\right\rangle\left\langle C_{1}^{*}>_{\Omega_{1}}\right)-\varepsilon \frac{\left.\partial\left(\theta_{2}^{*}<C_{2}^{*}\right\rangle\right)}{\partial t^{*}}+O\left(\varepsilon^{2}\right)\right.$

From Equation (70), it can be seen that convection is dominant at the macroscopic scale. Dispersion as well as the mass exchange term, which is reponsible for tailing (non Fickian behaviour), appear as correction at the order $\varepsilon$.

The equation governing the transport in the micro-porosity domain is obtained by adding Equations (56) and (57) multiplied by $\varepsilon$. This leads to

$\frac{\partial\left(\theta_{2}^{*} C_{2}^{*}\right)}{\partial t^{*}}=\varepsilon \nabla_{y} \cdot\left(\boldsymbol{D}_{2}^{*} \nabla_{y} C_{2}^{*}\right)$ in $\Omega_{2}$.

Finally, the macroscopic model in the dimensional form can be written as follows:

$\phi_{\mathrm{M}} \frac{\partial\left(\theta_{\mathrm{M}} C_{\mathrm{M}}\right)}{\partial t}=\nabla_{X} \cdot\left(\boldsymbol{D}_{\mathrm{disp}} \nabla_{X} C_{\mathrm{M}}\right)-\nabla_{X} \cdot\left(\boldsymbol{V} C_{\mathrm{M}}\right)-\frac{\partial\left\langle\theta_{\mathrm{m}} C_{\mathrm{m}}\right\rangle}{\partial t}$

where

$\frac{\partial\left\langle\theta_{\mathrm{m}} C_{\mathrm{m}}\right\rangle}{\partial t}=\frac{1}{|\Omega|} \int_{\Omega_{\mathrm{m}}} \frac{\partial\left(\theta_{\mathrm{m}} C_{\mathrm{m}}\right)}{\partial t} d \Omega$

where the notations " $\mathrm{M}$ " and " $\mathrm{m}$ " denote the macro- and micro-porosity domain respectively . $C_{\mathrm{M}}$ in Equation (72) is the average solute concentration in the liquid phase in the macroporosity domain with respect to the volume of the macro-porosity. $\left\langle C_{\mathrm{m}}\right\rangle$ is the average local solute concentration in the liquid phase in the micro-porosity domain, with respect to the total volume of the period. $\boldsymbol{V}$ is the Darcy unsaturated water flow velocity.

Equation (72) is coupled with the solute transport equation in the micro-porosity domain

$\frac{\partial\left(\theta_{\mathrm{m}} C_{\mathrm{m}}\right)}{\partial t}=\nabla_{X} \cdot\left(\boldsymbol{D}_{\mathrm{m}} \nabla_{X} C_{\mathrm{m}}\right)$ in $\Omega_{\mathrm{m}}$

and the condition at the interface $\Gamma$

$C_{\mathrm{M}}=C_{\mathrm{m}}$ on $\Gamma$.

The model (72)-(75) is the double-porosity convection-dispersion model (DP-DC). It consists of two equations for two concentration fields, which are coupled by an exchange term. This model describes the solute transport in the local non equilibrium conditions.

Note that the effective parameter of the model is an anisotropic dispersion tensor $\boldsymbol{D}_{\text {disp, }}$. It can be calculated from the solution of the local boundary problem (51)-(53) using the local 
dispersion tensor $\boldsymbol{D}_{1}$ which is locally velocity-dependent. The experimental validation of this model is presented in sections 4 and 5 .

\section{DISPERSION EXPERIMENTS IN THE DOUBLE-POROSITY MEDIUM}

\subsection{Materials and experimental set-up}

\subsubsection{Double-porosity physical model}

The DP physical model was used to perform dispersion experiments in fully controlled laboratory conditions. The same model was previously used to study the unsaturated water flow model $[24,25,26]$. It is built from two porous materials: the Hostun sand HN 38 and the solidified clayey spheres (radius, $R=3.2 \times 10^{-3} \mathrm{~m}$ ). The sand was considered as the macroporosity domain, whereas the clayey spheres are the micro-porosity domain. The laser granulometry of the sand showed a uniform grain size distribution with the mean grain size of $162 \mu \mathrm{m}$. Its composition was mainly quartz $\left(\mathrm{SiO}_{2}>97.5 \%\right)$. The sand was mechanically compacted and its porosity was kept constant and equal to $n_{\mathrm{M}}=0.400$ in all experiments. The mercury porosimetry test gave an average porosity of the solidified clay $n_{\mathrm{m}}=0.348$ (against 0.343 measured by gamma rays attenuation technique [38]) and the mean pore size of $0.7 \mu \mathrm{m}$. The scanning electronic microscopy of the clay indicated that it contains $\mathrm{Si}, \mathrm{Al}, \mathrm{Ca}$ and traces of some other metals ( $\mathrm{K}, \mathrm{Fe}, \mathrm{Mg}$ and $\mathrm{Ti}$ ). The contrast between average pores sizes of the two components of the double-porosity medium was about 1:200. It was shown that such contrast is sufficient to observe the double porosity effects [27].

For dispersion experiments a Plexiglas column (total height, $L_{\mathrm{t}}=0.6 \mathrm{~m}$ and inner diameter, $d$ $=0.06 \mathrm{~m}$ ) was used. The soil column was filled up alternatively with sand and spheres, layer by layer to obtain the medium length $L=0.5 \mathrm{~m}$, (about 110 layers) (Figure 2). The volumetric fractions of the two materials were almost the same. We controlled the mass and the mechanical energy of compaction. Such rigorous protocol allowed us to obtain columns containing three-dimensional periodic (cubic centered) microstructure $\ell=12.96 \times 10^{-3} \mathrm{~m}$ (Figure 2). It can be noted that the ratio of the characteristic lengths (microscopic / macroscopic) was about $1 / 50$.

\subsubsection{Experimental set-up}

The soil column was fixed in the mobile rig, equipped with a gamma ray $\left({ }^{241} \mathrm{Am}\right)$ attenuation device [38], (Figure 3). The latter allows measuring local porosity or water content changes along the vertical axis of the column during the experiment. As a supply system for steadystate unsaturated water flow and tracer displacement, two volumetric pumps of the range 0$500 \mathrm{~cm}^{3} / \mathrm{h}$ (Amersham Bioscience Pump P-500), were used. These pumps also were employed together with a pressure sensor Rosemount (0-3.5 bar) to determine the hydraulic conductivity at saturation. We obtained the following values of $K_{s}: 2.87 \times 10^{-5} \mathrm{~m} / \mathrm{s}$ and $1.96 \times 10^{-8} \mathrm{~m} / \mathrm{s}$ for the Hostun sand and the solidified clay respectively (Figure 4). Solution of $\mathrm{NaCl}$ was used as the passive tracer. The salt was dissolved in the water which was purified by means of a degassing/stirring machine. The $\mathrm{NaCl}$ concentration in the effluent was calculated from the density measurements (densimeter Anton Paar ${ }^{\circledR}, \mathrm{mP}$ 200). This apparatus measures simultaneously the temperature and the fluid density, with a precision of $1 / 100$ and $5 / 100000$, respectively. 


\subsection{Dispersion tests: experimental procedure and program}

The column dispersion tests were carried out in two steps. During the first step the permanent unsaturated water flow was established. The constant flow was imposed from the bottom of the column until the steady-state regime was reached. The cumulative outflow flux was measured by a balance and the recorded data were transferred to a computer via datalogger (Campbell Scientific Ltd CR 10X). At this stage, the water content was measured by gamma rays attenuation technique. Thus, the unsaturated permanent condition was obtained when two criteria were verified: $\theta<\theta_{\mathrm{S}}\left(\theta_{\mathrm{S}}\right.$ : water content at saturation) and $F_{\text {inlet }}=F_{\text {oulet }}$ (cumulative flux at inlet and outlet of the column). The second step consisted in tracer transport. The fluid displacement was started by switching the four-lines valve in order to connect the $\mathrm{NaCl}$ solution pump (5 g/l) and to close the water pump. Two different ways (pulse and stepwise) of tracer injection were used in the experiment, so that the valve was switched again or not according to pulse or stepwise condition. The time evolution of the $\mathrm{NaCl}$ concentration in the effluent (Breakthrough Curve, BTC) was recorded every $300 \mathrm{~s}$ by the densimeter connected to the computer to store all data. The dispersion test was considered ended when densimeter signal was stable, and the water content profile was measured again and compared with the initial one.

A series of such $\mathrm{NaCl}$ dispersion experiments were conducted in both DP medium (Hostun sand and clayey spheres) and SP medium (Hostun sand alone). This allowed us to compare the dispersive behavior between them and also to identify independently the required parameters of the sand as a constituent of the DP medium. Table 1 presents the main characteristics of the experiments and the experimental program. Note that the same Darcy velocity value was used in all experiments.

In this paper three dispersion tests in the double-porosity medium and two tests in the sand are presented.

\subsection{Results}

Figure 5 presents for Test 1, 4 and 5, the measured water content profiles corresponding to the unsaturated permanent water flow. Unfortunately, we could not measure the water content profiles of Test 2 and 3 because of the dysfunction of the mobile part of the gamma rays device. It can be observed that the distribution of the water content was not uniform and was more scattered in the DP medium (Test 1). The average value of all water content measurements in the sand were $\theta=0.355$ and $\langle\theta\rangle=0.313$ in the DP medium. In the numerical simulations (section 5), uniform water content and constant average values were used.

In order to obtain the $\mathrm{NaCl}$ concentration history at the outlet of the column, we calculated the relative concentration $C_{\mathrm{M}} / C_{0}(t)\left(C_{\mathrm{o}}\right.$ is the reference concentration) from the measured density variations with time. It can be noted that the relation between density and concentration revealed to be linear in our experimental conditions. The breakthrough curves (BTC) were obtained after filtering the data and they are shown in Figure 6a, for the stepwise-type boundary condition (single- and double-porosity medium) and in Figure 6b, for the pulse-type (double-porosity medium only).

The tracer mass balance was verified and reported in Table 2. Note that the mass balance was computed using the curves of the density in the effluent. The difference between the in/out 
salt mass and the mass present in the column was of the order of $3 \%$ (except $9.6 \%$ for Test 1 ). We checked that no reactive phenomena occurred in the column due to the composition of the two materials. Therefore, it is believed that the differences in the masses in the mass balance may come either from the fact that the materials were not perfectly washed between successive dispersion tests, or/and from the precision of the water content measurement by gamma rays technique.

Figure 6 shows the effect of the double structure, like early breakthrough and tailing indicating a non- Fickian behaviour. More detailed qualitative analysis of the obtained BTCs was presented in $[39,40]$. In section 5 these results are used to validate the double-porosity theoretical model developed by homogenization, Equations (72)-(75).

\section{EXPERIMENTAL VALIDATION OF THE MODEL}

The dispersion experiment data were used to validate the theoretical model obtained by homogenization. In order to be able to perform the numerical calculations, the theoretical model was implemented for the case of the experiment, namely 1D physical problem taking place in the DP medium presenting a 3D microstructure (Figure 2). This implementation was done using the Comsol Multiphysics ${ }^{\circledR}$ finite element code which is well suited for solving the coupled problems (flow and transport, micro- and macro- porosity domains). The validation process was carried out in two stages: calibration and validation. During the calibration stage the effective parameter of the model (dispersion coefficient) and some other parameters were determined. In the validation stage, the parameters were kept unchanged to simulate two independent dispersion experiments.

\subsection{Numerical implementation of the double-porosity dispersion model}

\subsubsection{Formulation of the problem}

The macroscopic dispersion model (72)-(75) corresponding to the 1D experimental conditions (Figure 2 and Figure 3), is written as follows:

$$
\begin{aligned}
& \phi_{\mathrm{M}} \frac{\partial\left(\theta_{\mathrm{M}} C_{\mathrm{M}}\right)}{\partial t}=\frac{\partial}{\partial z}\left(D_{\text {disp }} \frac{\partial C_{\mathrm{M}}}{\partial z}-\langle v\rangle C_{\mathrm{M}}\right)-Q\left(C_{\mathrm{m}}, t\right), \\
& \frac{\partial\left(\theta_{\mathrm{m}} C_{\mathrm{m}}\right)}{\partial t}=D_{\mathrm{m}}\left(\frac{\partial^{2} C_{\mathrm{m}}}{\partial r^{2}}+\frac{2}{r} \frac{\partial C_{\mathrm{m}}}{\partial r}\right), \\
& C_{\mathrm{M}}=C_{\mathrm{m}} \text { on the interface, }
\end{aligned}
$$

where $\mathrm{z}$ and $r$ [L] are the vertical and radial coordinate respectively. The source term, $Q$ $\left[\mathrm{ML}^{3} \mathrm{~T}^{-1}\right]$, expresses the solute exchange between the two sub-domains and is defined by:

$$
Q\left(C_{\mathrm{m}}, t\right)=\frac{1}{|\Omega|} \int_{\Gamma} D_{\mathrm{m}} \frac{\partial C_{\mathrm{m}}}{\partial r} d \Gamma .
$$

The initial and boundary conditions are as follows: 
i) Initial condition: $t \leq 0, C_{\mathrm{M}}=C_{\mathrm{m}}=0$;

ii) Boundary condition for Equation (76):

- For stepwise test (Test 1 and 2):

$z=0, t>0:\langle v\rangle C_{\mathrm{M}}-D_{\text {disp }}\left(\theta_{\mathrm{M}}\right) \frac{\partial C_{\mathrm{M}}}{\partial z}=\langle v\rangle C_{0}$,

$z=L, t>0: D_{\text {disp }} \frac{\partial C_{\mathrm{M}}}{\partial z}=0$

where $C_{0}$ is the applied concentration.

- For pulsewise test (Test 3):

$$
\begin{aligned}
& z=0,0<t \leq t_{0}:\langle v\rangle C_{\mathrm{M}}-D_{\text {disp }}\left(\theta_{\mathrm{M}}\right) \frac{\partial C_{\mathrm{M}}}{\partial z}=\langle v\rangle C_{0}, \\
& z=0, t>t_{0}:\langle v\rangle C_{\mathrm{M}}-D_{\text {disp }}\left(\theta_{\mathrm{M}}\right) \frac{\partial C_{\mathrm{M}}}{\partial z}=\langle v\rangle C_{i},
\end{aligned}
$$

where $C_{\mathrm{i}}=0$ (water used for tracer displacement).

$z=L, t>0: D_{\text {disp }} \frac{\partial C_{\mathrm{M}}}{\partial z}=0$

where $t_{0}[\mathrm{~T}]$ is a pulse duration of the tracer solution injected in the medium.

iii) Boundary condition for Equation (77):

at the interface

$r=0, t>0: C_{\mathrm{M}}(z)=C_{\mathrm{m}}(r)$,

at the center of the sphere

$r=R, t>0: D_{\mathrm{m}} \frac{\partial C_{\mathrm{m}}}{\partial r}=0$

\subsubsection{Strategy of the numerical implementation}

The numerical implementation concerned the macroscopic one dimensional equation (76) coupled with a series of one dimensional problems (77), together with initial (80) and boundary conditions (81)-(85) and (86)-(87). The implementation was performed using the commercial Comsol Multiphysics ${ }^{\circledR}$ code. The strategy required the domain transformations as follows [41]: 
- Equation (76) is to be solved in the 1D domain of the homogenized medium. The dimension of the domain corresponds to the hight of the column,

- A series of 1D problems in the micro-porosity domains (Equations (77)) were transformed into an anisotropic diffusion problem in the 2D rectangular domain. This new domain has the dimensions of the hight of the column and the radius of the spheres. Diffusion was allowed in the radius direction only.

- The coupling was obtained by imposing the concentration equality condition (78) and by defining that the source term in Equation (76) is equal to the average flux along the micro-macro interface, Equation (79).

The schematic illustration of the numerical implementation is presented in Figure 7. The macroscopic domain was uniformly discretized into 100 elements $\left(\Delta z=5 \times 10^{-3} \mathrm{~m}, 101\right.$ nodes $)$. There was initially 19 elements $\left(\Delta r=0.17 \times 10^{-3} \mathrm{~m}, 20\right.$ nodes $)$ for the direction $r$. Because the size of the domain in $r$ direction is short $\left(r=3.2 \times 10^{-3} \mathrm{~m}\right)$ in comparison with the dimension of the macroscopic domain, $L=0.5 \mathrm{~m}$, the microscopic domain was finely re-meshed by using the re-scaling factor $L / r$. In this manner, the number of elements (1483 triangular elements) was increased. The mesh was finer close to the interface. It was checked that the results were mesh independent. The time step varied in the range $0.001 \mathrm{~s}-0.1 \mathrm{~s}$.

\subsection{Comparison between numerical simulations and experimental measurements}

In order to validate the theoretical model, we carried out the numerical simulations of the experiments and we compared the results with the observations. This process consists of two stages: calibration and validation. Test 1 (stepwise-type) for the calibration stage and Test 2 (stepwise-type) and Test 3 (pulsewise-type) for the validation stage were used.

\subsubsection{Model calibration}

The model (76)-(78) was calibrated by fitting the dispersion coefficient $D_{\text {disp }}\left(\theta_{\mathrm{M}}\right)$ to the experimental breakthrough curve of the Test 1 . The other model parameters were either known or estimated via an independent analysis. Concerning the water contents $\theta_{M}$ and $\theta_{m}$, it was assumed that $\theta_{M}$ in the macro-porosity domain was the same as in pure sand tests (Test 4 and 5) because both SP and DP experiments were performed at the same unsaturated flow conditions. Therefore, we have $\theta_{\mathrm{M}}=0.355$. On the other hand, because the experiment was performed in the conditions close to saturation, we considered that $\theta_{m}$ in the micro-porosity domain is equal to $\theta_{m \mathrm{~s}}(=0.343)$. Taking into account these two values, the average water content in the double-porosity medium was calculated as $\langle\theta\rangle_{\text {cal }}=\phi_{\mathrm{M}} \theta_{\mathrm{M}}+\phi_{\mathrm{m}} \theta_{\mathrm{m}}=0.348$. Note that this value is higher than the one measured by gamma rays technique $\left(\langle\theta\rangle_{\text {mes }}=\right.$ 0.313 ) with $10 \%$ difference (which corresponds to the precision of mass balance for Test 1 , see Table 2). This may be explained by the fact that the saturation of the DP medium slightly increased with long duration of the experiment. The diffusion coefficient $D_{\mathrm{m}}\left(\theta_{\mathrm{m}}\right)$ in the microporosity domain (solidified clay) was taken from the literature for brick material at saturation Alh [42], $\left(D_{\mathrm{m}}=7.345 \times 10^{-11} \mathrm{~m}^{2} / \mathrm{s}\right)$. Fitting of the dispersion coefficient of the DP medium $D_{\text {disp }}\left(\theta_{\mathrm{M}}\right)$ was achieved by taking the dispersion coefficient in pure sand tests (Test 4 and 5) as the initial value. The latter value, equal to $D_{\mathrm{M}}=1.50 \times 10^{-9} \mathrm{~m}^{2} / \mathrm{s}$, was obtained by inverse analysis of the experiments in sand, using the CXTFIT code [43]. The calibrated dispersion coefficient in the DP medium was found $D_{\text {disp }}=2.374 \times 10^{-8} \mathrm{~m}^{2} / \mathrm{s}$. 
Figure 8 shows a very satisfying agreement between the simulated and measured values of concentration time evolution at the outlet of the column for Test 1 . Note that some measurement points were removed from the BTC for the sake of better visualization. Statistics given in Table 3 confirm the accuracy of the fit. Finally, it has to be pointed out that the dispersion coefficient of the DP medium was found to be greater than the one of the SP medium (sand) for the same Darcy velocity condition. That confirms the exprimental results published in the literature for highly heterogeneous soils

\subsubsection{Model validation}

Since all experiments were performed in the same flow condition, the model parameters obtained from Test 1 can be employed to simulate two other independent experiments with different boundary conditions: Test 2 (stepwise-type) and Test 3 (pulsewise-type with pulse duration $t_{0}=4,620 \mathrm{~s}$ ). Figures 9 and 10 give the calculated and measured concentration evolution with time at the outlet of the column are presented. Excellent agreement are observed and confirmed by the statistics in Table 3 for Test 2 and 3. It can be concluded that the developed model was successfully validated by a very good description of all the features of the BTCs observed in the experiments, particularly their early advance and tailing.

\section{DISCUSSION}

The results of the dispersion experiment were used to analyze some assumptions defining the domain of validity of the model.

Firstly, let us verify the condition defining the double porosity medium which is

$$
\frac{K_{2}}{K_{1}}=O\left(\varepsilon^{2}\right)
$$

where the small parameter is $\varepsilon=\ell / L=2.6 \times 10^{-2}$. For the experimental condition close to saturation, the coefficients $K$ can reasonably be considered as the saturated hydraulic conductivity. Hence, we have

$$
\frac{K_{2}}{K_{1}}=\frac{1.96 \times 10^{-8}}{2.86 \times 10^{-5}}=6.9 \times 10^{-3}
$$

meaning that the assumption is verified since:

$$
17.6 \times 10^{-6}<\frac{K_{2}}{K_{1}}=O\left(\varepsilon^{2}\right)<<2.6 \times 10^{-2} \text {. }
$$

Three assumptions concerning the homogenization of the transport problem have to be verified:

i) First, we assumed that the characteristic time of the process is the time of convection through the macro-porosity domain. From Figure 6 we can see that this time is of the order $O\left(10^{5} \mathrm{~s}\right)$ which corresponds to the time of the transport by convection in the macro-porosity domain at the scale $L$ 
$t_{c}=\frac{L \theta_{1 c}}{v_{1 c}}=\frac{0.51 \times 0.355}{3.44 \times 10^{-6} / 0.49}=25789 \mathrm{~s}$.

ii) Second condition concerns the ratio of the diffusion/dispersion coefficients:

$$
G=\frac{D_{2 c}}{D_{1 c}}=O\left(\varepsilon^{2}\right)
$$

with $D_{2 \mathrm{c}}=7.345 \times 10^{-11} \mathrm{~m}^{2} / \mathrm{s}$ and $D_{1 \mathrm{c}}=2.374 \times 10^{-8} \mathrm{~m}^{2} / \mathrm{s} / 0.490$ (estimated value). These lead to:

$$
\frac{D_{2 c}}{D_{1 c}}=\frac{7.345 \times 10^{-11}}{2.374 \times 10^{-8} / 0.490}=1.5 \times 10^{-3}
$$

So, we can see that the condition is satisfied

$6.7 \times 10^{-4}<\frac{D_{2 c}}{D_{1 c}}=O\left(\varepsilon^{2}\right)<<2.6 \times 10^{-2}$.

iii) Finally, the Péclet number calculated as:

$$
P e_{1 \ell}=\frac{\ell v_{1 c}}{D_{1 c}}=\frac{12.96 \times 10^{-3} \times 3.44 \times 10^{-6} / 0.49}{2.374 \times 10^{-8} / 0.49}=1.8
$$

falls within the domain of validity since

$$
2.6 \times 10^{-2}<P e_{1 \ell}=O\left(\varepsilon^{0}\right)<<62.5
$$

for the experiments which were carried out.

Following this analysis, it can be concluded that the domain of validity of the model applied to the experimental dataset was respected and was based on physically justified assumptions.

\section{CONCLUSIONS}

Using the asymptotic homogenization method, we derived a double-porosity dispersionconvection model, consisting of two coupled equations at macro- and microscopic scale. The existence of two concentration fields provides evidence of the local non equilibrium condition during the transient phase of the process, and resulting in early breakthrough and tailing curves, reported in the literature as preferential transport. The derivation was based on physical arguments and estimates of the non dimensional parameters, which was confirmed by dispersion experiments on a physical model of double-porosity. It is interesting to notice that we obtain a double-porosity dispersion model for the case of a large contrast of water hydraulic conductivities at saturation for the macro- and micro-porosity domains (of the order $\varepsilon^{2}$ ), while the ratio of the diffusion coefficients at saturation in the two domains is of the order $\varepsilon^{0}(1: 14)$. It means that the contrast of diffusion coefficients is not needed to observe the 
double-porosity effects in the solute transport. Such situation seems to correspond to the physical reality of a wide class of geomaterials.

The theoretical model has one effective parameter: the dispersion tensor. It depends on the local transport characteristics in the macro-porosity domain and the microstructure of the medium. The homogenization approach allows us to better understand the relation between the microstructure and the macroscopic dispersive properties. It has to be pointed out that dispersion tensor is strongly velocity dependent. It can be determined experimentally from the steady-state dispersion experiments.

The model was numerically implemented by using the finite element code Comsol Multiphysics ${ }^{\circledR}$. A particular strategy of the implementation was proposed to enable the twoscale coupled computations, in the case of one dimensional physical process taking place in a porous medium presenting three dimensional (cubic centered) microstructure.

The obtained double-porosity model was experimentally validated. The experiments were carried out in a physical model, consisting of periodic assemblage of clayey spheres and fine sand carefully packed in a vertical soil column. The experiments were carried out in unsaturated conditions close to saturation. It was shown that the model reproduced with an excellent accuracy a series of dispersion experiments of $\mathrm{NaCl}$ tracer in controlled laboratory conditions. Of particular interest are the results showing the effect of local non-equilibrium prevailing in a double-porosity medium on the early advance and tailing of the breakthrough curves as compared to what it expected by considering single homogeneous porous material.

As for all models developed by upscaling approaches, the model implies the existence of scale separation. Moreover, the domain of validity of the dispersion model, defined by means of non dimensional parameters, has to be verified.

It is believed that the proposed modeling approach can be extended for the case of reactive solute transport. Other physical phenomena conducting to hydro-chemico-mechanical couplings can also be foreseen.

\section{ACKNOWLEGEMENTS}

The present study is a part of the $\mathrm{PhD}$ thesis of the first author. The research was supported by the CNRS, France through a BDI-PED doctoral fellowship granted to the first author and in the framework of the GDR "Hydrodynamique et Transferts dans les Hydrosystèmes Souterrains". All the financial supports are gratefully acknowledged. 


\section{REFERENCES}

1. Šimůnek J, Jarvis N, van Genuchten M.T., Gardenas A. Review and comparison of models for describing non-equilibrium and preferential flow and transport in the vadose zone. Journal of Hydrology 2003; 272: 14-35.

2. Gerke H.H. Preferential flow description for structured soils. Journal of Plant Nutrition and Soil Science 2006; 169: 382-400.

3. Köhne J.M., Köhne S, Šimůnek J. A review of model applications for structured soils: A) Water flow and tracer transport. Journal of Contaminant Hydrology 2009; 104: 4-35.

4. Barenblatt G., Zheltov I., Kochina I. Basic concepts in the theory of seepage of homogeneous liquids in the fissured rocks. Journal of Applied Mathematics 1960; 24(5):1286-1303.

5. van Genuchten M.T., Wierenga P.J. Mass transfer studies in sorbing porous media : I). analytical solutions. Soil Science Society of America Journal 1976; 40: 473-80.

6. Gerke H.H, van Genuchten M.T. A dual-porosity model for simulating the preferential movement of water and solutes in structured porous media. Water Resources Research 1993; 29: 305-319.

7. Pôt V., Šimůnek J., Benoit P, Coquet Y., Yra A., Martinez-Cordon M.J. Impact of rainfall intensity on the transport of two herbicides in undisturbed grassed filter strip soil cores. Journal of Contaminant Hydrology 2005; 81: 63-88.

8. Jarvis N. The MACRO model (version 3.1). Technical description and sample simulation. Report and dissertation 1994; Dept. Soil Science, Sweden University of Agricultural Sciences: 51.

9. Gerke H.H., van Genuchten M.T. Macroscopic representation of structural geometry for simulating water and solute movement in dual-porosity media. Advances in Water Resources 1996; 19:343-357.

10. Sanjuan B. et al. Tracer testing of the geothermal heat exchanger at Soultz-sous-Forêts (France) between 2000 and 2005. Geothermics 2006; 35: 622-653.

11. Gentier S., Rachez X., Trần Ngọc T. D., Peter-Borie M., Souque C. 3D flow modelling of the medium-term circulation test performed in the deep geothermal site of Soultz-sous-Forêts (France). Proc. World Geothermal Congress, Bali, Indonesia, 2010. (in press)

12. Schwartz R.C., Juo A.S.R., McInnes K.J. Estimating parameters for a dual porosity model to describe non-equilibrium, reactive transport in a fine-textured soil. Journal of Hydrology 2000; 229: 149-167.

13. Gerke H.H., Köhne J.M. Dual-permeability modeling of preferential bromide leaching from a tile-drained glacial till agricultural field, Journal of Hydrology 2004; 289: 239- 257. 
14. Katterer T., Schmied B., Abbaspour K.C., Schulin R. Single and dual porosity modelling of multiple tracer transport through soil columns : effects of initial moisture and mode of application. European Journal of Soil Science 2001; 52: 25-36.

15. Köhne S., Lennartz B., Köhne J., Šimůnek J. Bromide transport at a tile-drained field site: experiment, and one- and two- dimensional equilibrium and non-equilibrium numerical modeling. Journal of Hydrology 2006; 321: 390-408.

16. Šimůnek J., van Genuchten M.T. Modeling non-equilibrum flow and transport processes using Hydrus. Vadose Zone Journal 2008; 7: 782-797.

17. Ahmadi A., Quintard M., Whitaker S. Transport in chemically and mechanically heterogeneous porous media. V: Two-equation model for solute transport with adsorption. Advances in Water Resources 1998; 22: 59-86.

18. Quintard M., Whitaker S. Two-phase flow in heterogeneous porous media: The method of large scale averaging. Transport in Porous Media 1988, pp. 357-413.

19. Chella R., Lasseux D., Quintard M. Multiphase, multicomponent fluid flow in homogeneous and heterogeneous porous media. Revue de l'Institut Francais du Pétrole 1998 ; 53 (3) : 335-346.

20. Peszynska M., Showalter R. Multi scale elliptic-parabolic systems for flow and transport. Electronic Journal of Differential Equations 2007; 147: 1-30.

21. Hornung U. Homogenization of miscible displacement in unsaturated aggregated soils. Dynamical systems, St. Petersburg 1991; 143-158.

22. Richards L. Capillary conduction of liquids through porous medium. Physics 1931; 1: 318-333.

23. Mikelic A., Rosier C. Modeling solute transport through unsaturated porous media using homogenization I. Computational \& Applied Mathematics 2004; 23 (N. 2-3): 195-211.

24. Lewandowska J., Szymkiewicz A., Gorczewska W., Vauclin M. Infiltration in a doubleporosity medium: Experiments and comparison with a theoritical model. Water Resources Research 2005; 41(2): 1-14.

25. Lewandowska J., Trần Ngọc T. D., Vauclin M., Bertin H. Water drainage in double porosity soils: Experiments and micro-macro modelling. ASCE Journal of Geotechnical and Geoenvironmental Engineering 2008; 134(2): 231-243.

26. Szymkiewicz A., Lewandowska J., Angulo-Jaramillo R., Butlanska J. Two scale modeling of unsaturated water flow in a double-porosity medium under axi-symmetric conditions. Canadian Geotechnical Journal 2008; 45: 238-251.

27. Lewandowska J., Szymkiewicz A., Burzynski K., Vauclin M. Modeling of unsaturated water flow in double - porosity soils by the homogenization approach. Advances in Water Resources 2004; 27: 283-296. 
28. Bear J. On the tensor form of dispersion in porous media. Journal of Geophysical Research 1961; 66: 1185-97.

29. Auriault J. Heterogeneous medium. is an equivalent macroscopic description possible ? International Journal of Engineering Science 1991; 29:785-795.

30. Gaudet J. P., Jégat H., Vachaud G., Wierenga P. J. Solute transfer, with exchange between mobile and stagnant water, through unsaturated sand. Soil Science Society of America 1977, 41: 665-671.

31. Zinn B., L., Meigs C., Harvay C. F., Haggerty R., Peplinski W. J., Von Schwerin C. F. Experiments visualization of solute transport and mass transfer processes in two dimensional conductivity fields with connected regions of high conductivity. Environmental Science and Technology 2004, 38: 3916-3926.

32. Tejchman M. Solute transport through single and dual porosity media. Mathematical modelling by homogenisation and column experimental investigations, PhD Thesis 2004, Université Joseph Fourier-Grenoble, France.

33. Cherblanc F., Ahmadi A., Quintard M. Two-medium description of dispersion in heterogeneous porous media: Calculation of macroscopic properties. Water Resources Research 2003, 39 (6): 1559-1579.

34. Bensoussan A., Lions J., Papanicolaou G. Asymptotic analysis for periodic structures 1978, North-Holland.

35. Sanchez-Palencia E. Non-homogeneous media and vibration theory. Vol. 127 of Lecture Notes in Physics 1980; Berlin, Springer-Verlag.

36. Auriault J., Lewandowska J. Diffusion/adsorption/advection macrotransport in soils. European Journal of Mechanics - A/Solids 1996; 15(4): 681-704.

37. Auriault J., Adler P. Taylor dispersion in porous media : Analysis by multiple scale expansions. Advances in Water Resources 1995 ; 18: 217-226.

38. Gharbi D., Bertin H., Omari A. Use of gamma rays attenuation technique to study colloids deposition in porous media. Experiments in Fluids 2004; 37(5), 665-672.

39. Trần Ngọc T. D, Lewandowska J., Bertin H. Etude expérimentale de la dispersion dans un milieu à double-porosité en condition saturée et insaturée. Congrès Français de Mécanique 2007; Grenoble, 6 (CD-rom support).

40. Trần Ngọc T. D., Lewandowska J., Bertin H., Vauclin M., Do Hong L. C. Dispersion in double-porosity unsaturated medium: From experiment toward modeling by homogenization. Proc. $4^{\text {th }}$ Biot Conference on Poromechanics 2009, H. I. Ling, A. Smyth et R. Betti, eds., DEStech Publication Inc, Pennsylvania, 306-311. 
41. Trần Ngọc T. D. Transport de solutés dans un milieu à double-porosité non saturé. Modélisation par homogénéisation et application. Ph.D thesis 2008 ; Université Joseph Fourier-Grenoble, $185 \mathrm{p}$ (in French).

42. Ahl J. Salt diffusion in brick structures. Journal of Materials Science 2003; 38: 20552061.

43. Toride N., Leij F.J, van Genuchten M.T. The CXTFIT code for estimating transport parameters from laboratory or field tracer experiments. version 2.1. Salinity laboratory Research Report 1999; U. S. Salinity, Riverside, CA, 137p. 


\section{LIST OF TABLES}

Table 1. Main characteristics of the experiments. L is the medium length; $\phi \mathrm{M}$ and $\phi \mathrm{m}$ are the volumetric fractions of the macro- and micro-porosity domains, respectively; $\mathrm{nM}$ and $\mathrm{nm}$ are the porosities of the macro- and micro-porosity domains measured by gravity and gammametry, respectively; $<\theta>$ is the average volumetric water content of the doubleporosity domain measured by gamma attenuation technique; $\langle\mathrm{v}>$ is the imposed Darcy velocity; $\mathrm{C} 0$ is the tracer concentration applied at the entry of the column; DP and SP stand for double-porosity and single-porosity (pure sand), respectively.

Table 2. Solute mass balance measured in the five tests. M1 and M2 are the input and ouput salt masses; M0 is the salt mass present in the medium; DP and SP stand for double- and single-porosities, respectively.

Table 3. Statistics of the model efficiency for Test 1 (calibration), Test 2 and Test 3 (validation). SSQ is the objective fonction; R is the correlation coefficient; RMSE is the root mean square error (see defintions in [41]). 
TWO-SCALE MODELING OF SOLUTE DISPERSION

\begin{tabular}{|lccccccccc|}
\hline & $\begin{array}{c}L \\
{[\mathrm{~m}]}\end{array}$ & $\phi_{M}$ & $\phi_{m}$ & $n_{M}$ & $n_{m}$ & $<\theta>$ & $<\nu>$ & Type & $\begin{array}{c}C_{0} \\
{[\mathrm{~g} / \mathrm{l}]}\end{array}$ \\
\hline Test 1 (DP) & 0.510 & 0.490 & 0.510 & 0.378 & 0.343 & 0.313 & $3.44 \times 10^{-6}$ & Step & 5 \\
Test 2 (DP) & 0.469 & 0.483 & 0.517 & 0.386 & 0.343 & & $3.44 \times 10^{-6}$ & Step & 5 \\
Test 3 (DP) & 0.469 & 0.483 & 0.517 & 0.386 & & & $3.44 \times 10^{-6}$ & Pulse & 5 \\
Test 4 (SP) & 0.503 & & & 0.404 & & 0.354 & $3.44 \times 10^{-6}$ & Step & 5 \\
Test 5 (SP) & 0.502 & & & 0.400 & & 0.355 & $3.44 \times 10^{-6}$ & Step & 5 \\
\hline
\end{tabular}

Table 1. Main characteristics of the experiments. $L$ is the medium length; $\phi_{M}$ and $\phi_{m}$ are the volumetric fractions of the macro- and micro-porosity domains, respectively; $n_{M}$ and $n_{m}$ are the porosities of the macro- and micro-porosity domains measured by gravity and gammametry, respectively; $\langle\theta\rangle$ is the average volumetric water content of the double-porosity domain measured by gamma attenuation technique; $\langle v\rangle$ is the imposed Darcy velocity; $C_{0}$ is the tracer concentration applied at the entry of the column; DP and SP stand for double-porosity and single-porosity (pure sand), respectively. 
T. D. TRAN NGOC ET AL.

\begin{tabular}{|lccccc|}
\hline & $\mathrm{M}_{1}[\mathrm{~g}]$ & $\mathrm{M}_{2}[\mathrm{~g}]$ & $\mathrm{M}_{1}-\mathrm{M}_{2}[\mathrm{~g}]$ & $\mathrm{M}_{0}[\mathrm{~g}]$ & $\Delta \mathrm{M}[\%]$ \\
\hline Test 1 (DP) & 6.038 & 3.518 & 2.520 & 2.277 & 9.631 \\
Test 2 (DP) & 5.177 & 2.910 & 2.267 & 2.057 & 4.263 \\
Test 3 (DP) & 0.225 & 0.226 & & & 0.400 \\
Test 4 (SP) & 4.054 & 1.496 & 2.596 & 2.530 & 1.492 \\
Test 5 (SP) & 4.185 & 1.630 & 2.556 & 2.459 & 3.779 \\
\hline
\end{tabular}

Table 2. Solute mass balance measured in the five tests. $M_{1}$ and $M_{2}$ are the input and ouput salt masses; $\mathrm{M}_{0}$ is the salt mass present in the medium; DP and SP stand for double- and single-porosities, respectively. 


\begin{tabular}{|lccc|}
\hline & $S S Q$ & $R^{2}$ & $R M S E$ \\
\hline Test 1 & 0.028 & 0.9981 & $3.5 \times 10^{-4}$ \\
Test 2 & 0.002 & 0.9999 & $2.4 \times 10^{-5}$ \\
Test 3 & 0.016 & 0.9820 & $3.5 \times 10^{-5}$ \\
\hline
\end{tabular}

Table 3. Statistics of the model efficiency for Test 1 (calibration), Test 2 and Test 3 (validation). SSQ is the objective fonction; $R$ is the correlation coefficient; $R M S E$ is the root mean square error (see defintions in [41]). 


\section{LIST OF FIGURES}

Figure 1: Macro- and microscopic scales in the double-porosity periodic medium.

Figure 2: Microstructure (periodic) of the double-porosity medium used in the experiments (units in $\mathrm{mm}$ ) (The small figure reprinted from Proceedings of the Fourth Biot Conference on Poromechanics IV, 2009. Lancaster, PA: DEStech Publications, Inc. with permission).

Figure 3: Scheme of the set-up used for the dispersion experiments in the unsaturated doubleporosity medium. Gamma rays attenuation device (radioactive 241Am source, scintillator, photomultiplicator mounted on a mobile platform); pumps (Amersham Bioscience P-500); $\mathrm{NaCl}$ tracer dissolved in the water purified by inverse osmosis (Milli-ro 3 Plus); Densimeter (Anton Paar, mP 200) measuring the density of tracer in the effluent from the porous medium Figure 4: Measured hydraulic conductivity at saturation Ks of sand, double-porosity medium (DP) and solidified clay deduced from the slope of the relation between Darcy flux q and pressure gradient $\Delta \mathrm{P} / \mathrm{L}$.

Figure 5: Measured volumetric water content profiles by gamma rays attenuation technique in the double-porosity (Test 1 ) and in the simple-porosity (Test 4 and 5) media.

Figure 6: Measured breakthrough curves: a) stepwise-type for the double-porosity medium (Test 1 and 2) and for the simple-porosity medium (Test 4 and 5); b) pulsewise-type for the double-porosity medium (Test 3 ).

Figure 7: a) Scheme of homogenized double-porosity medium; b) General principle of the numerical implementation of the double-porosity model (scheme not to scale).

Figure 8: Calculated (bold line) and measured (symbols) breakthrough curves for Test 1: calibration stage (stepwise-type boundary) (This figure reprinted from Proceedings of the Fourth Biot Conference on Poromechanics IV, 2009. Lancaster, PA: DEStech Publications, Inc. with permission).

Figure 9: Simulated (bold line) and measured (symbols) breakthrough curves for Test 2: validation stage (stepwise-type boundary) (This figure reprinted from Proceedings of the Fourth Biot Conference on Poromechanics IV, 2009. Lancaster, PA: DEStech Publications, Inc. with permission).

Figure 10: Simulated (bold line) and measured (symbols) breakthrough curves for Test 3: validation stage (pulsewise-type boundary) (This figure reprinted from Proceedings of the Fourth Biot Conference on Poromechanics IV, 2009. Lancaster, PA: DEStech Publications, Inc. with permission). 


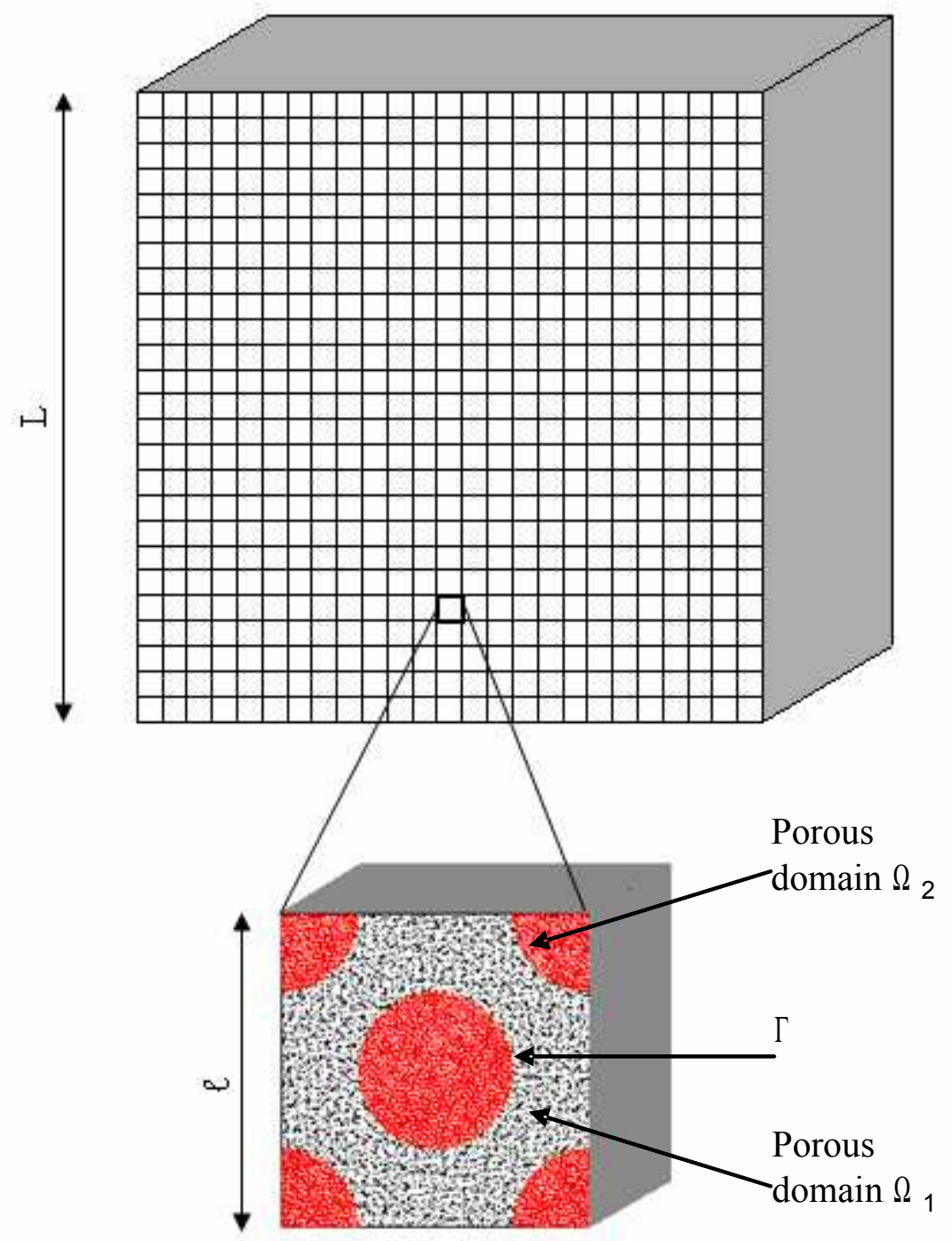

Figure 1: Macro- and microscopic scales in the double-porosity periodic medium. 

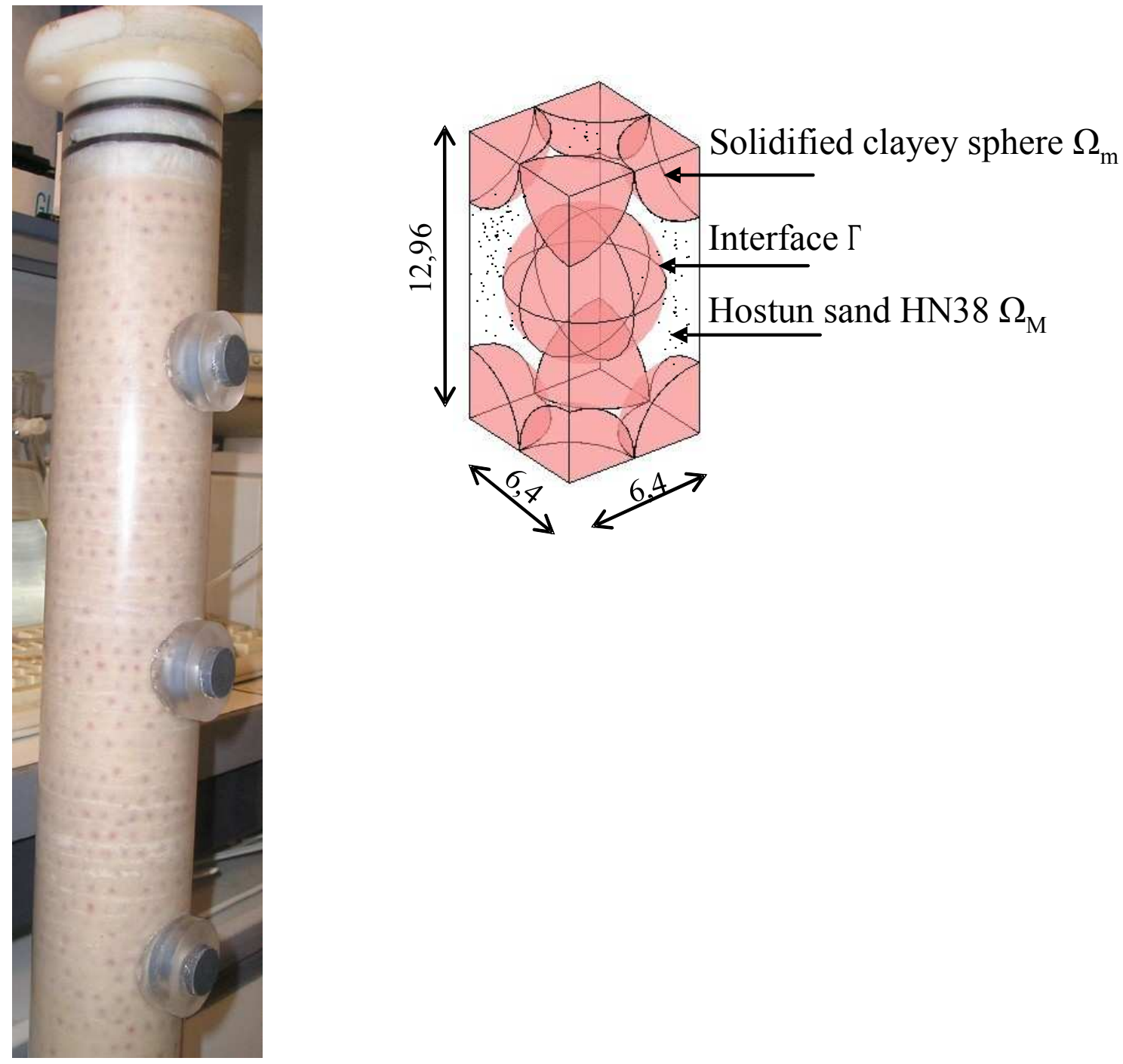

Figure 2: Microstructure (periodic) of the double-porosity medium used in the experiments (units in $\mathrm{mm}$ ) (The small figure reprinted from Proceedings of the Fourth Biot Conference on Poromechanics IV, 2009. Lancaster, PA: DEStech Publications, Inc. with permission). 


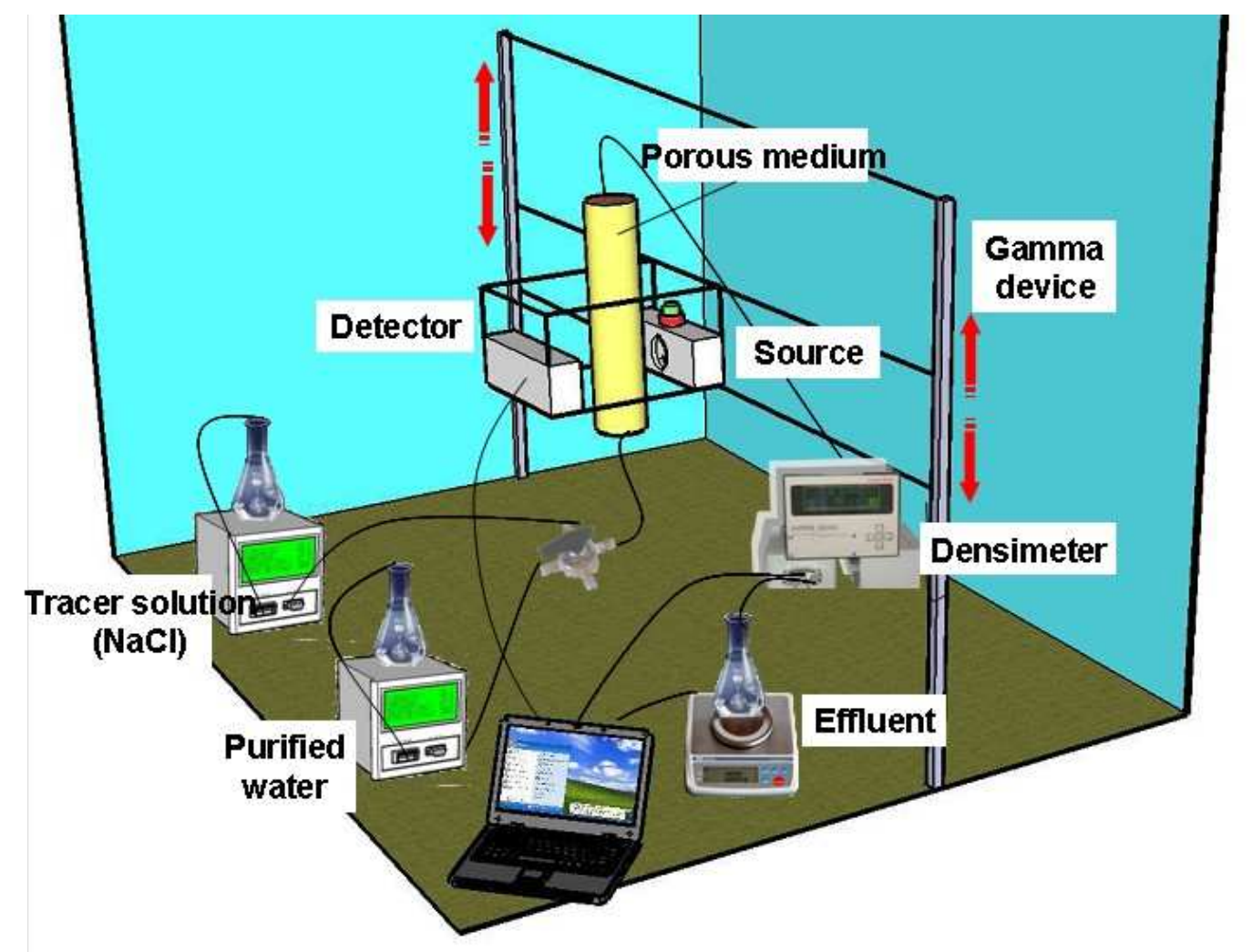

Figure 3: Scheme of the set-up used for the dispersion experiments in the unsaturated doubleporosity medium. Gamma rays attenuation device (radioactive ${ }^{241} \mathrm{Am}$ source, scintillator, photomultiplicator mounted on a mobile platform); pumps (Amersham Bioscience P-500); $\mathrm{NaCl}$ tracer dissolved in the water purified by inverse osmosis (Milli-ro 3 Plus); Densimeter (Anton Paar, mP 200) measuring the density of tracer in the effluent from the porous medium 


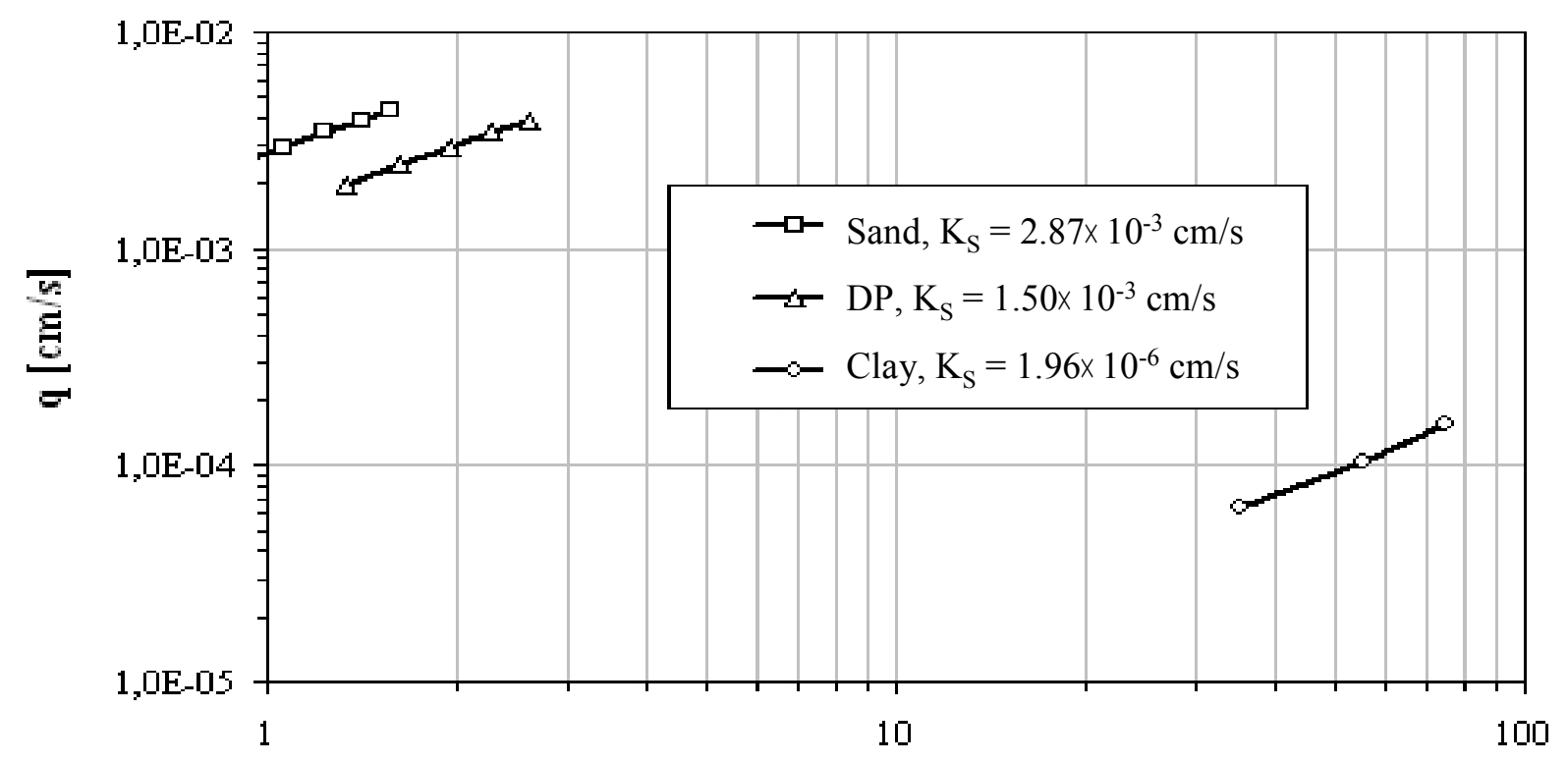

$\Delta \mathbf{P} / \mathbf{L}[-]$

Figure 4: Measured hydraulic conductivity at saturation $\mathrm{K}_{\mathrm{s}}$ of sand, double-porosity medium (DP) and solidified clay deduced from the slope of the relation between Darcy flux $q$ and pressure gradient $\Delta \mathrm{P} / \mathrm{L}$. 


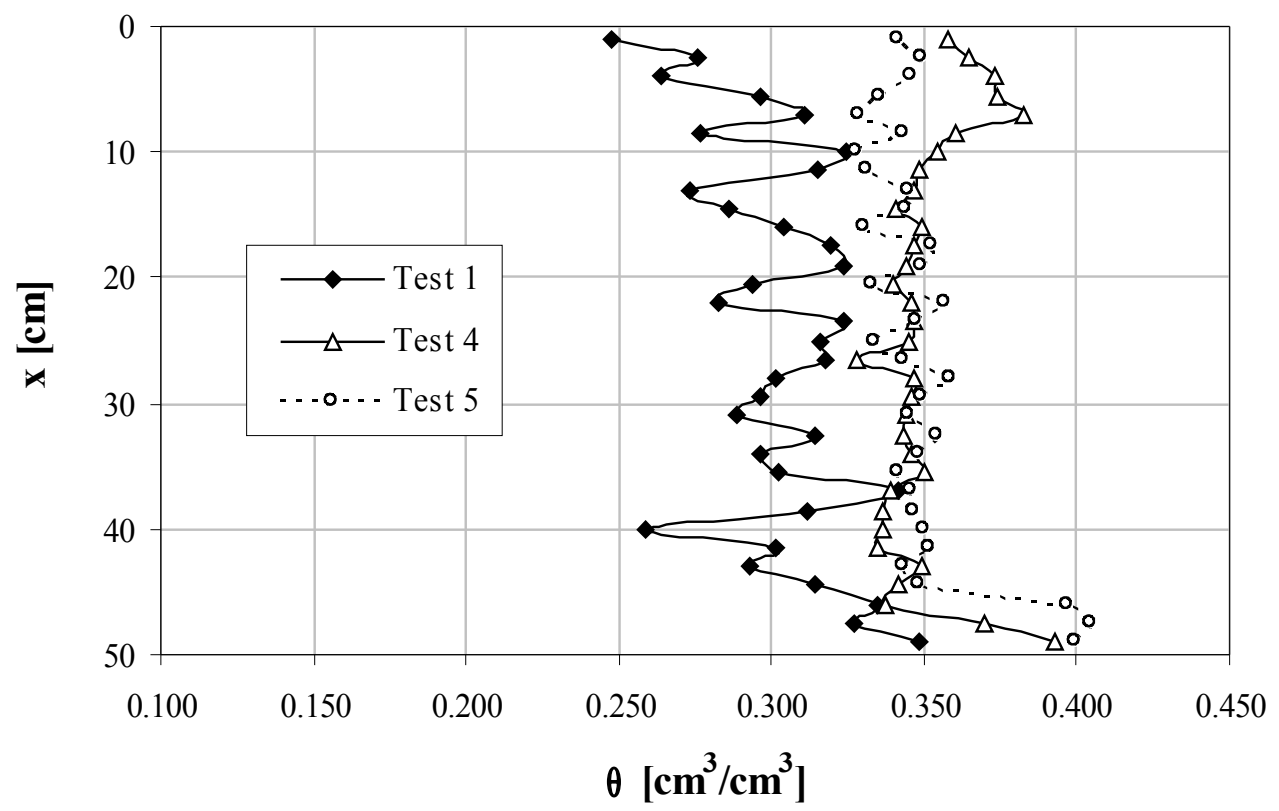

Figure 5: Measured volumetric water content profiles by gamma rays attenuation technique in the double-porosity (Test 1) and in the simple-porosity (Test 4 and 5) media. 

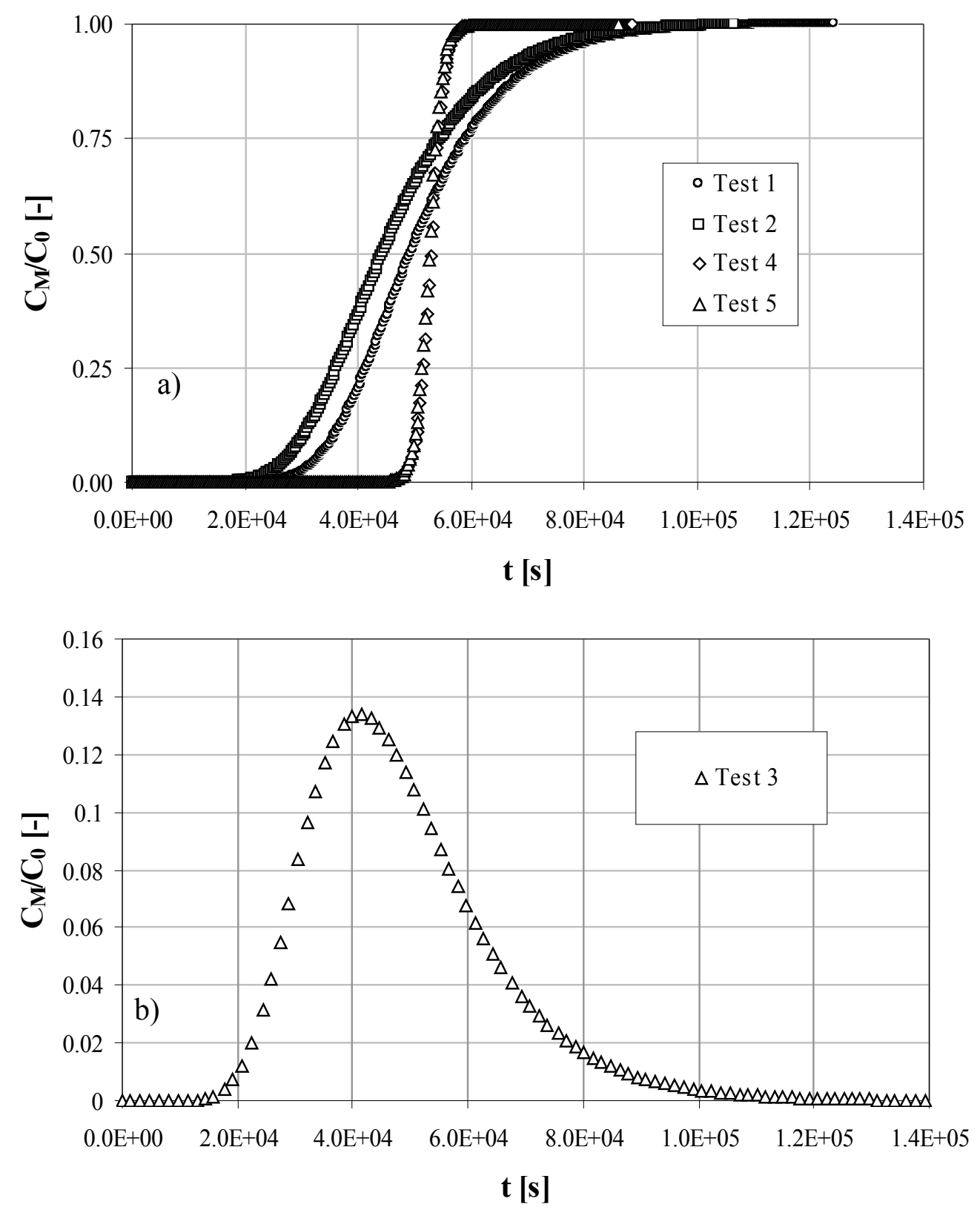

Figure 6: Measured breakthrough curves: a) stepwise-type for the double-porosity medium (Test 1 and 2) and for the simple-porosity medium (Test 4 and 5); b) pulsewise-type for the double-porosity medium (Test 3 ). 


\section{TWO-SCALE MODELING OF SOLUTE DISPERSION}

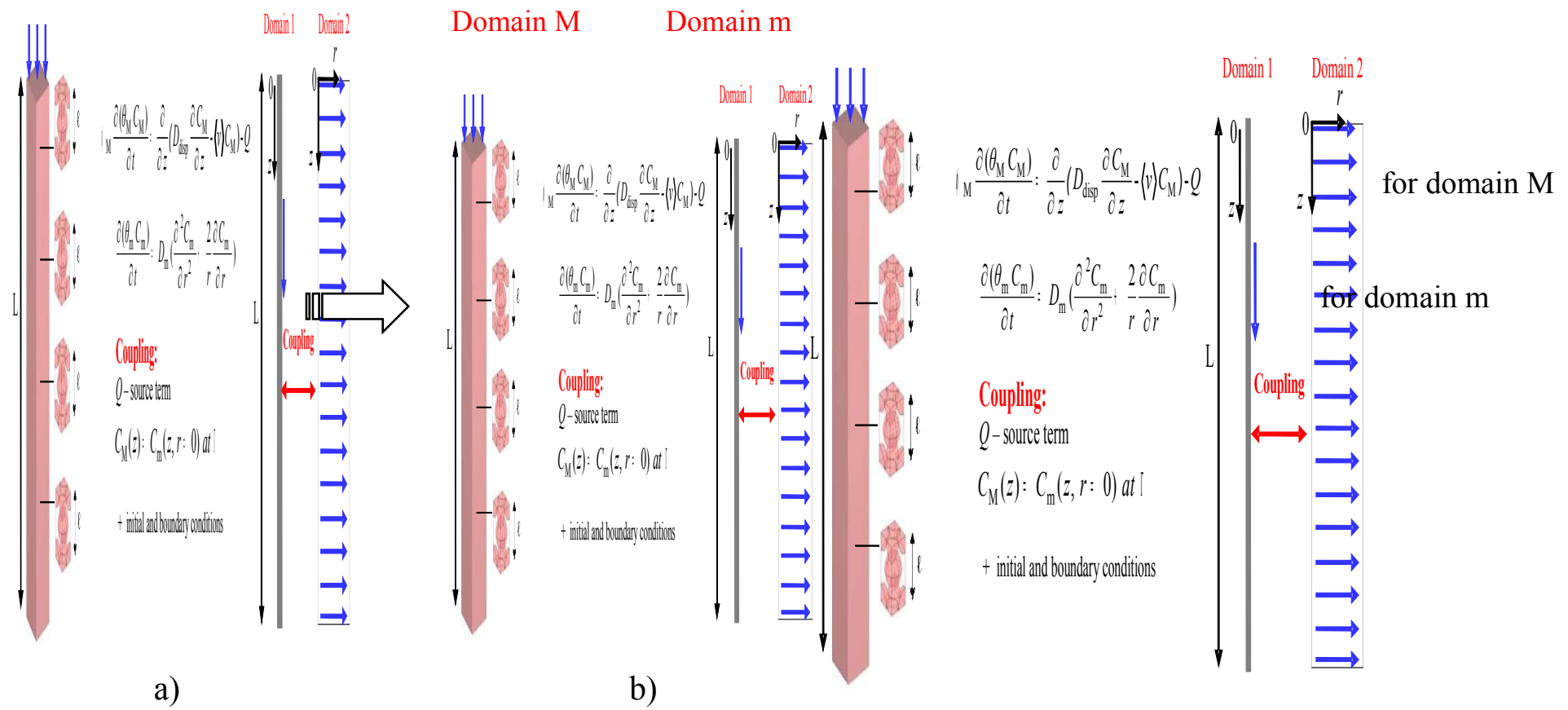

Figure 7: a) Scheme of homogenized double-porosity medium; b) General principle of the numerical implementation of the double-porosity model (scheme not to scale). 
T. D. TRAN NGOC ET AL.

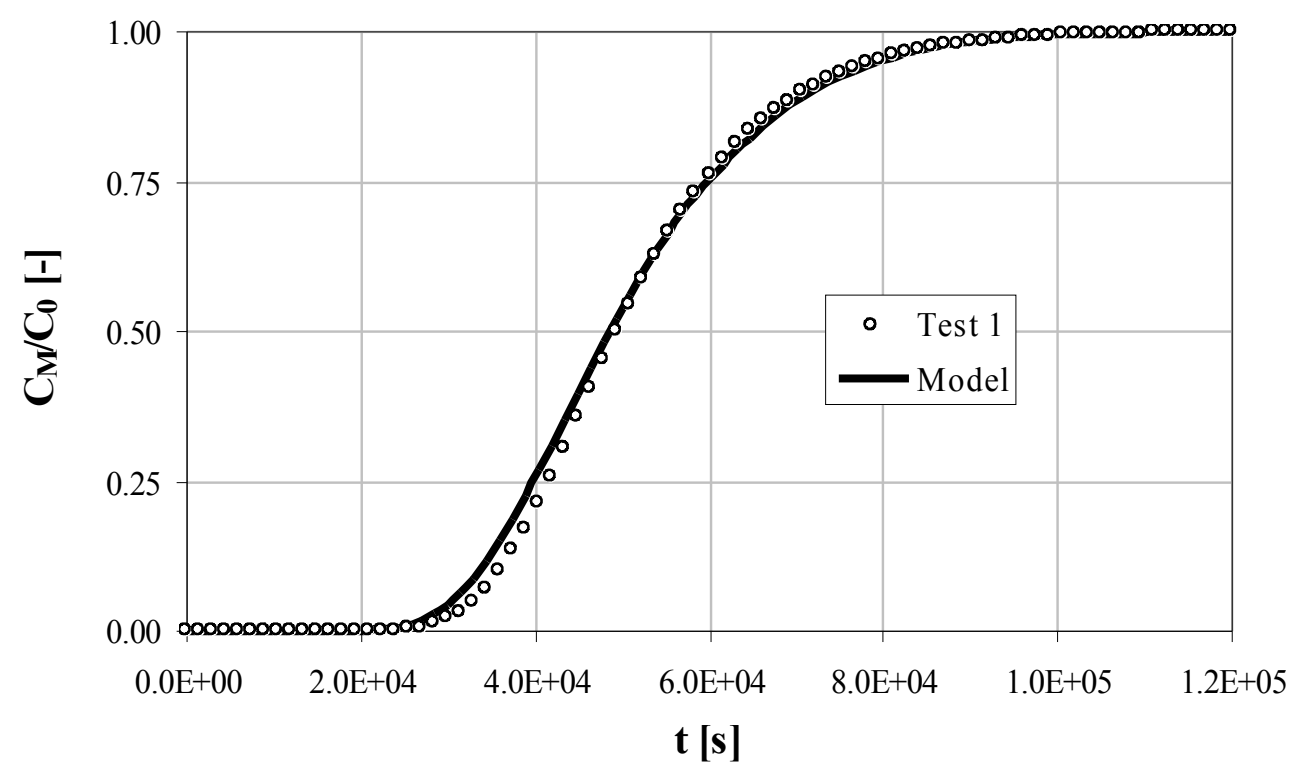

Figure 8: Calculated (bold line) and measured (symbols) breakthrough curves for Test 1: calibration stage (stepwise-type boundary) (This figure reprinted from Proceedings of the Fourth Biot Conference on Poromechanics IV, 2009. Lancaster, PA: DEStech Publications, Inc. with permission). 


\section{TWO-SCALE MODELING OF SOLUTE DISPERSION}

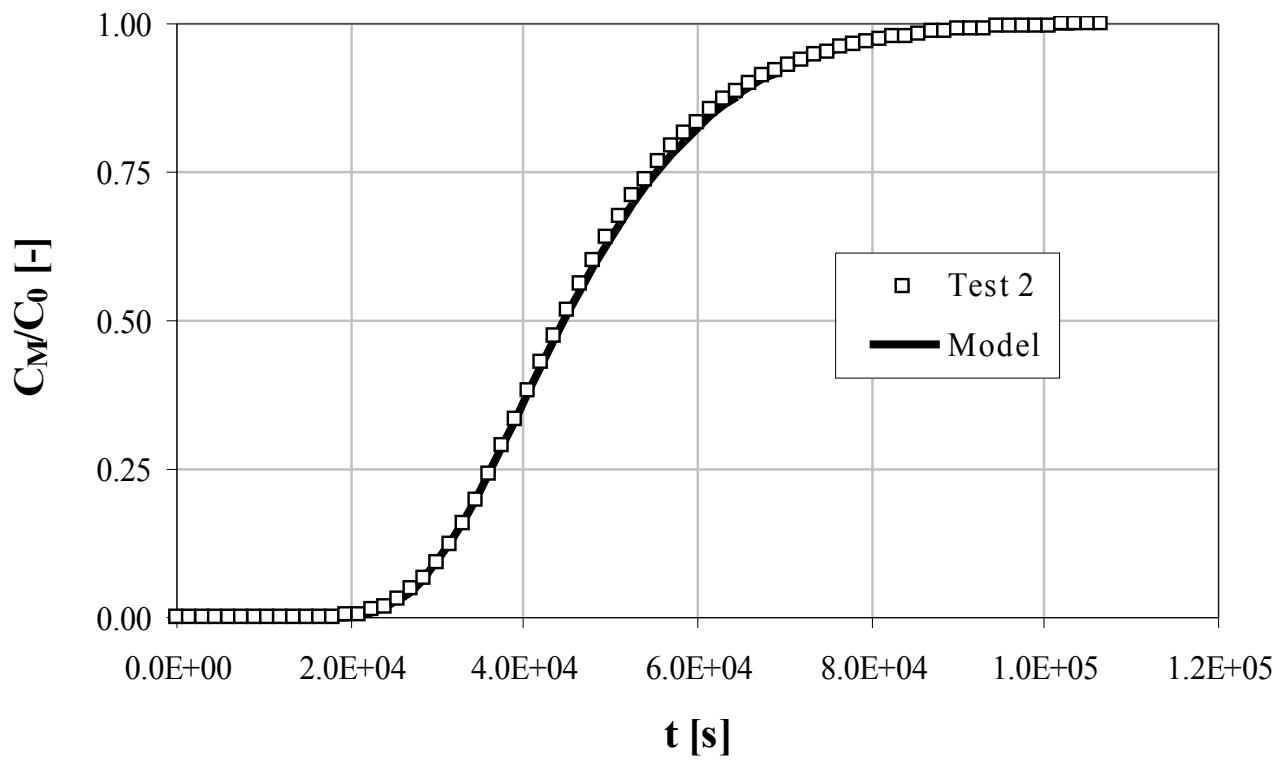

Figure 9: Simulated (bold line) and measured (symbols) breakthrough curves for Test 2: validation stage (stepwise-type boundary) (This figure reprinted from Proceedings of the Fourth Biot Conference on Poromechanics IV, 2009. Lancaster, PA: DEStech Publications, Inc. with permission). 
T. D. TRAN NGOC ET AL.

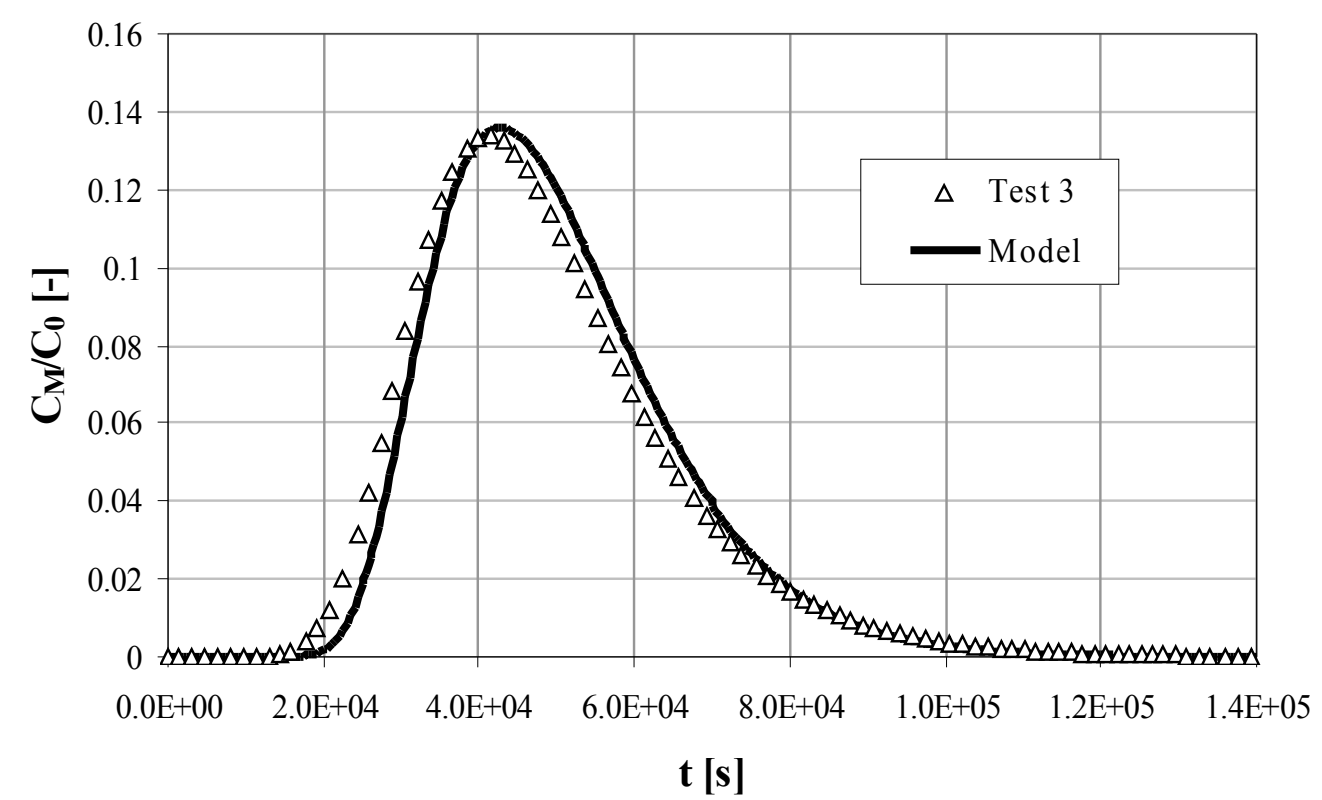

Figure 10: Simulated (bold line) and measured (symbols) breakthrough curves for Test 3: validation stage (pulsewise-type boundary) (This figure reprinted from Proceedings of the Fourth Biot Conference on Poromechanics IV, 2009. Lancaster, PA: DEStech Publications, Inc. with permission). 